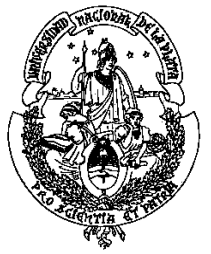

Maestría en Economía

Facultad de Ciencias Económicas

Universidad Nacional de La Plata

TESIS DE MAESTRIA

ALUMNO

Leonardo Peñaloza Pacheco

TITULO

El Impacto del Éxodo Migratorio de Venezolanos sobre los Salarios reales en Colombia

DIRECTOR

María Irene Brambilla y María Laura Alzúa (codirectora)

FECHA DE DEFENSA

$5 / 10 / 2019$ 


\title{
El impacto del éxodo migratorio de venezolanos sobre los salarios reales en Colombia
}

\author{
Leonardo Peñaloza Pacheco ${ }^{1}$ \\ UNLP
}

Febrero 2019

\begin{abstract}
Resumen
El objetivo del presente trabajo consiste en estimar el efecto causal del éxodo migratorio de venezolanos hacia Colombia a partir del año 2016 sobre el salario real colombiano. Se explota como estrategia de identificación la apertura de las fronteras entre Colombia y Venezuela en el segundo semestre del 2016, luego de un año de estar cerradas producto de una crisis política entre los dos países. Se estima que, de acuerdo con datos de la Unidad Administrativa Especial de Migración Colombia y el Registro Administrativo de Migrantes Venezolanos en Colombia, postapertura de las fronteras entre los dos países, el flujo migratorio de venezolanos hacia Colombia representó aproximadamente un incremento de entre el $10 \%$ y el $15 \%$ de la Población Económicamente Activa de los departamentos fronterizos de Colombia como La Guajira y Norte de Santander en los cuales se basa el análisis. Implementando las metodologías de diferencias en diferencias y Método de Control Sintético los resultados indican que el incremento en la oferta laboral en estas regiones, producto del flujo migratorio, generó una caída promedio en el salario real horario de aproximadamente 9.7\%. Esta disminución del salario real parecería ser mayor para los hombres en comparación a las mujeres. Además, se evidencia una mayor caída en el salario real de las personas con menor nivel de calificación y en condiciones de informalidad laboral.
\end{abstract}

JEL: J31, J61, F22.

Palabras Claves: Migración, Salarios, Colombia, Venezuela

\footnotetext{
${ }^{1}$ El presente trabajo constituye mi Tesis de Maestría en Economía de la FCE-UNLP realizada bajo la dirección de las profesoras Irene Brambilla y María Laura Alzúa. A ellas agradezco enormemente toda la orientación y el apoyo a lo largo de este proceso y de mi formación como profesional. También agradezco los comentarios recibidos por Julián Martinez Correa, Carlo Lombardo, Leopoldo Tornarolli, Matías Ciaschi, Mariana Marchionni y los participantes del Seminario de Avance de Tesis y de la LIII Reunión Anual de la Asociación Argentina de Economía Política. Cualquier error u omisión es de mi exclusiva responsabilidad. Correo electrónico: leopacheco93@gmail.com
} 


\section{INTRODUCCIÓN}

Uno de los principales temas a los cuales se ha dedicado la economía laboral es a analizar las consecuencias económicas de los flujos migratorios en los mercados laborales de los países receptores de migrantes. Desde hace más de 25 años, en la literatura ha habido intensos debates dedicados a estimar si hay un impacto significativo de un incremento en la oferta laboral producto de flujos migratorios importantes sobre las variables del mercado de trabajo, principalmente sobre el salario real en las regiones en las cuales aumenta considerablemente la oferta de trabajo.

De acuerdo con Borjas (2017), la relevancia práctica de este tipo de análisis radica en que permite direccionar las decisiones de política económica tomadas por los policy makers para mitigar los efectos negativos de los flujos migratorios, por supuesto, si estos existiesen.

La mayoría de los trabajos vinculados con el análisis del impacto de los flujos migratorios sobre el mercado de trabajo toman como variación exógena la llegada de migrantes, en general refugiados, producto de alguna crisis o evento extraordinario en sus países o regiones de origen (Ver por ejemplo Card, 1990; Hunt, 1992; Carrington y De Lima, 1994; Borjas, 2017; entre otros).

La idea principal en torno a la cual han girado muchos de los análisis realizados en la literatura es que, dada una demanda de trabajo con pendiente negativa, ceteris paribus, un shock muy fuerte en la oferta laboral vía un flujo migratorio, siguiendo los modelos canónicos de economía neoclásica, generaría una caída significativa en el salario real percibido por los trabajadores.

En el presente trabajo se explota como variación exógena del mercado de trabajo colombiano al flujo migratorio de venezolanos desde Venezuela hacia Colombia a partir de la segunda mitad del año 2016, fecha en la cual, tal y como se explicará más adelante con mayor detalle, hubo un éxodo migratorio significativo de venezolanos producto de la apertura de las fronteras entre estos dos países luego de aproximadamente un año de estar cerradas.

Con base en este shock exógeno de oferta de trabajo, se analizará el impacto en términos salariales que se presentó a partir del año 2016 en las regiones fronterizas colombianas las cuales se vieron más afectadas por este fenómeno.

Se propondrán estrategias empíricas basadas en las metodologías de diferencias en diferencias y control sintético que permitan estimar el efecto causal sobre los salarios agregados y garantizar la robustez de los resultados, con sus respectivos efectos heterogéneos sobre subgrupos poblacionales por sexo, nivel de calificación y formalidad laboral.

La literatura en este tema toma como bases distintos casos en diferentes economías a nivel internacional y los resultados que se han obtenido distan profundamente de converger hacia un efecto único esperado. Hunt (1992), por ejemplo, evidencia una caída en el salario anual de aproximadamente un $1.3 \%$ en 1967 producto de la repatriación de personas de Argelia en el mercado laboral francés. Friedberg (2001), por su parte, analiza el efecto de la migración generada entre 1990 y 1994 desde la extinta Unión Soviética hacia Israel y, empleando variables instrumentales, no evidencia un efecto significativo sobre los outcomes del mercado laboral de Israel.

Por otro lado, Malaeb y Wahba (2018) analizan el efecto del flujo de refugiados provenientes de Siria sobre el mercado laboral de migrantes en Jordania evidenciando una caída del salario total post-flujo migratorio (Para una revisión más amplia de la literatura ver, por ejemplo, Angrist y Kuegler, 2003; Alix-Garcia y Saah, 2009; Ruiz y Vargas-Silva, 2016; Tumen, 2016; entre otros).

Adicionalmente, quizás uno de los trabajos pioneros y más influyente en esta materia es el de Card (1990). En su trabajo de 1990, David Card intenta estimar el efecto sobre el mercado laboral 
de la llegada de los marielitos ${ }^{2}$ a Miami en Estados Unidos, flujo migratorio que incrementó la oferta laboral, según el autor, en aproximadamente un $7 \%$. De acuerdo con las estimaciones de Card (1990), el flujo migratorio de los marielitos no tuvo un efecto significativo sobre el mercado laboral de Miami, argumentando la capacidad de la economía de Miami para absorber rápidamente a la oferta laboral y evitar, de esta manera, un ajuste en el corto plazo de los salarios reales.

A partir del análisis de Card (1990) han surgido diversos trabajos que han buscado refutar - principalmente en términos metodológicos - los resultados obtenidos mediante análisis desagregados de la población afectada por la migración. Borjas (2003 y 2017) argumenta que trabajadores con niveles educativos similares pero con diferentes niveles de experiencia no son sustitutos perfectos. Por lo tanto, este tipo de análisis se debería basar en subgrupos definidos en términos de educación y de experiencia laboral.

Otra de las lecciones que sugiere Borjas (2017) se vincula con lo fundamental que resulta la selección de los grupos de control para realizar las estimaciones del impacto de los flujos migratorios sobre las variables del mercado laboral en una región. ${ }^{3}$ De acuerdo a Borjas (2017), la selección de un grupo de control por parte del analista puede generar la obtención de resultados más fuertes o más débiles sobre las variables del mercado laboral, lo cual, por supuesto, influiría sobre las medidas a tomar por parte de los policy makers.

Con relación a la divergencia entre los resultados empíricos obtenidos en la literatura sobre el efecto de los flujos migratorios en el salario real, uno de los argumentos brindados por Dustmann et al. (2016) postula que, partiendo del modelo canónico neoclásico, cada uno de los trabajos intenta medir diferentes efectos. De acuerdo a Dustmann et al. (2016) las mediciones empíricas realizadas en la literatura estiman parámetros diferentes y no comparables del modelo canónico.

Con base en estas observaciones, Dustmann et al. (2016) dividen los resultados de la literatura en tres distintos enfoques: El primer enfoque, que según los autores es el empleado por Borjas (2003), se refiere al análisis a nivel nacional del efecto de la migración sobre los salarios entre grupos de distinto nivel educativo o experiencia. Un segundo enfoque, denominado enfoque espacial, explota las diferencias en términos de flujo migratorio entre distintas regiones (Ver, por ejemplo, Altonji y Card, 1991). Y, por último, proponen un tercer enfoque que se relaciona con una combinación de los dos anteriores (Ver por ejemplo Card, 1991).

En este trabajo se empleará el enfoque espacial dado que se presentarán las estimaciones del efecto del flujo migratorio de venezolanos sobre los salarios en Colombia, comparando entre regiones fuertemente afectadas por la migración y aquellas que no lo fueron. Adicionalmente, dado que se diferenciarán los efectos por grupos de nivel educativo, también se empleará el tercer enfoque que combina el enfoque espacial con el relacionado con los efectos por nivel de educación.

\footnotetext{
2 Se les conoce como marielitos a los cubanos que migraron desde la Isla de Cuba hacia el Estado de la Florida en Estados Unidos en 1980, en lo que se conoció como el Éxodo de Mariel. El flujo migratorio desde el Puerto de Mariel se produjo luego de un permiso especial otorgado por Fidel Castro para que los que lo desearan pudieran emigrar luego de tensiones políticas ocurridas en la isla previamente.

3 El comentario de Borjas (2017) se refiere principalmente sobre la selección ad-hoc del grupo de comparación realizada por Card (1990) para analizar las consecuencias en el mercado laboral del Éxodo de Mariel.
} 
Por otro lado, la literatura ha optado por estimar los efectos de un flujo migratorio mediante diferentes alternativas metodológicas ${ }^{4}$. Algunos trabajos ${ }^{5}$ como el de Card (1990), por ejemplo, optan por seleccionar un grupo de control de manera ad-hoc, esto es, discrecionalmente, para comparar la evolución de los salarios en las regiones afectadas por la migración con la evolución de los salarios en aquellas regiones seleccionadas ad-hoc.

Adicionalmente, existen trabajos que implementan el Método de Control Sintético (MCS) bajo el cual el grupo de control se construye con base en un desarrollo analítico que permite librar de arbitrariedad la selección del grupo de comparación y, de esta manera, conformar un grupo sintético a partir de diferentes ponderaciones de las regiones no afectadas por la migración.

El MCS es una técnica econométrica, introducida en el herramental analítico a partir de los trabajos de Alberto Abadie, implementada para analizar el impacto de hechos exógenos que afectan a una región en particular a partir de la comparación con las otras regiones no afectadas. (Ver, por ejemplo, Abadie y Gardeazabal, 2003; Abadie, Diamond y Hainmueller, 2010, 2015)

En términos de la economía laboral y vinculando el análisis con el efecto de los flujos migratorios sobre el salario real, Peri y Yasenov (2017) implementan el Método de Control Sintético propuesto por Alberto Abadie para revisar los resultados obtenidos por Card (1990). Los autores no evidencian ningún efecto negativo sobre los salarios de los trabajadores de baja calificación en Miami, producto del flujo migratorio de marielitos.

De acuerdo a Peri y Yasenov (2017), la ganancia en términos empíricos, de la implementación de MCS para este tipo de análisis sobre el mercado laboral, radica en que permite identificar un grupo de control "óptimo", elaborado a partir de la combinación lineal del resto de potenciales grupos de comparación disponibles, que minimice las diferencias en la variable de interés preshock migratorio entre las regiones afectadas por la migración y aquellas no influenciadas significativamente por ésta, eliminando la discrecionalidad en las estimaciones realizadas.

En este trabajo se optará por realizar un análisis empleando el enfoque de diferencias en diferencias implementado por Card (1990), seleccionando un grupo de control ad-hoc y, de manera concomitante, también se empleará el MCS para proporcionar mayor robustez a los resultados.

En las estimaciones presentadas se podrá observar que los resultados no difieren significativamente entre ambas metodologías y que, sin lugar a dudas, el flujo migratorio de venezolanos a partir del segundo semestre del año 2016 generó, en el corto plazo, una caída importante en el salario real de las regiones colombianas más afectadas por la migración de aproximadamente un $9.7 \%$ post apertura de las fronteras entre Colombia y Venezuela.

Este efecto parecería ser ligeramente superior para los hombres en comparación a las mujeres y mucho más fuerte para los trabajadores en situación de informalidad. Adicionalmente se mostrará que la caída en el salario real es más intensa cuanto menor es el nivel de calificación de los individuos.

Otra de las características novedosas de este trabajo radica en el hecho de que, en general, este tipo de análisis entre migración y mercado laboral se realizan a partir de un flujo migratorio masivo de un país en desarrollo a un país desarrollado, con lo cual la mano de obra que ingresa en el

\footnotetext{
4 Dustmann et al. (2016) brinda un recopilado de la literatura indicando las diferentes metodologías implementadas y los resultados principales de los trabajos más importantes. Ver también por ejemplo a Lozano y Steinberger (2010).

${ }^{5}$ En este trabajo nos enfocamos en aquellas investigaciones que han implementado metodologías de diferencias en diferencias. Existe también una amplia gama de análisis empíricos que han estimado los efectos de la migración sobre el mercado laboral implementando OLS, Variables Instrumentales, entre otros. (Ver Dustmann et al., 2016)
} 
mercado laboral del país receptor es de características muy diferentes a la mano de obra ya radicada.

En este trabajo, sin embargo, se analiza el impacto del flujo migratorio entre dos países que por su historia son muy similares en términos sociales y demográficos, con lo cual se esperaría una mayor sustituibilidad entre la mano de obra migrante y la radicada en la economía y, por lo tanto, un importante efecto negativo sobre los salarios.

El resto del trabajo se organiza de la siguiente manera: En la sección II se presentan los datos que permiten comprender y dimensionar el flujo migratorio hacia territorio colombiano proveniente de Venezuela a partir del segundo semestre del año 2016. En la sección III se proponen diferentes estrategias de identificación y metodologías para poder estimar el efecto causal del shock de oferta de trabajo sobre los salarios reales con sus correspondientes resultados. En la sección IV se estiman efectos heterogéneos del flujo migratorio sobre los salarios; se diferencian los resultados por sexo de la población, nivel de calificación y formalidad laboral. En la sección $V$ se elaboran ejercicios de robustez que permitan garantizar la interpretación causal de las estimaciones realizadas en este trabajo. Finalmente, en la sección VI se concluye.

\section{EL FLUJO MIGRATORIO DE VENEZOLANOS HACIA COLOMBIA}

\section{Crisis Política y Fronteriza entre Colombia y Venezuela}

En agosto del año 2015, debido a tensiones políticas y de seguridad entre Colombia y Venezuela, el Gobierno de Nicolás Maduro ordenó el estado de excepción durante 60 días en cinco municipios del Estado Táchira de Venezuela: Bolívar, Ureña, Junín, Capacho-Libertad y Capacho-Independencia. Posteriormente, fueron deportados más de 180 ciudadanos colombianos desde Venezuela, lo cual profundizó la crisis política y humanitaria generando que las fronteras entre los dos países se fueran gradualmente cerrando hasta abarcar todo el territorio fronterizo colombo-venezolano. Luego se tomaron similares medidas en Ayacucho, García de Hevia, Lobatera y Panamericano en Estado Táchira.

A principios de septiembre del 2015 se fueron cerrando las fronteras por órdenes del ejecutivo venezolano en los Estados Zulia y Apure, para finalmente a finales de septiembre del mismo año declarar las mismas medidas en los municipios de Atabapo, Atures, Autana, Maroa y Río Negro del Estado Amazonas de Venezuela el cual es fronterizo con la República de Colombia.

Luego de meses de negociaciones entre Nicolás Maduro y Juan Manuel Santos —presidente de Colombia desde el año 2010 hasta el año 2018- y tras diferentes acuerdos, se definió la reapertura de las fronteras entre colombianos y venezolanos el 13 de agosto del año 2016. La reapertura de las fronteras, sumado con la crisis económica y social presente en Venezuela, generó un éxodo masivo de venezolanos hacia tierras colombianas en el segundo semestre del año 2016 que se extendió a los semestres siguientes.

En la siguiente subsección se presentará información que permitirá, por un lado, dimensionar la magnitud de la migración venezolana hacia Colombia y, por otro lado, observar la manera en la cual claramente el flujo migratorio se intensificó precisamente a partir del segundo semestre del año 2016 en las regiones fronterizas colombianas con Venezuela; elemento fundamental para garantizar la validez de la estrategia de identificación propuesta en este trabajo. 


\section{Migración de Venezolanos a Colombia}

Para poder analizar el impacto sobre el salario de la migración venezolana en las regiones de Colombia, el análisis en este trabajo se basará en datos sobre los salarios horarios en términos reales y características laborales y sociodemográficas de los individuos de la Gran Encuesta Integrada de Hogares del Departamento Administrativo Nacional de Estadística (DANE) de Colombia.

En segundo lugar, los ingresos migratorios de venezolanos en Colombia por departamento de hospedaje se obtuvieron a partir de la información de la Unidad Administrativa Especial Migración Colombia (UAEMC) y, finalmente, la cantidad de venezolanos radicados irregularmente en territorio colombiano se obtuvo del Registro Administrativo de Migrantes Venezolanos (RAMV) ${ }^{6}$.

La ventaja principal de los datos del RAMV frente a los de la UAEMC es que los primeros nos indican la cantidad de venezolanos censados efectivamente radicados irregularmente en cada uno de los departamentos, con lo cual podremos controlar por posibles flujos interdepartamentales de los migrantes venezolanos una vez ingresaron en el país. Esto permite reforzar la estrategia de identificación propuesta en este trabajo y la selección de los departamentos en los cuales se esperaría una mayor caída en los salarios reales.

El periodo bajo estudio está comprendido entre el año 2014 y el primer semestre del año 2018. El análisis se hizo en periodos de frecuencia semestral tomando el ingreso salarial horario de los individuos en los meses de junio y diciembre de cada uno de los años, los cuales se consideraron como primer y segundo semestre de cada año, respectivamente.

Tal y como se observa en el Gráfico 1 la intensidad en el flujo migratorio y de migrantes radicados fue mucho mayor en los departamentos fronterizos del lado colombiano entre Colombia y Venezuela.

El ingreso migratorio acumulado entre el segundo semestre del año 2016 y el año 2017 es de $11.66 \%, 15.67 \%$ y $22.24 \%$ de la Población Económicamente Activa (PEA) para los departamentos de Norte de Santander, La Guajira y Guainía, respectivamente. Para Vichada y Arauca el flujo migratorio representa aproximadamente entre un $60 \%$ y un $200 \%$ de la PEA de estos departamentos, principalmente debido a que son departamentos de una baja población.

De acuerdo con el RAMV, el $18.6 \%$ de migrantes censados a nivel nacional se encuentran radicados en el Norte de Santander, mientras que el 16.92\% están en La Guajira, esto es, 82.286 y 74.874 personas, respectivamente. Estas cifras, tal y como se observa en la tercera columna de la Tabla A1 del Apéndice, equivalen aproximadamente al $10.26 \%$ y el $12.35 \%$ de la Población Económicamente Activa del Norte de Santander y La Guajira del año 2016, respectivamente?.

Con lo cual, incluso asumiendo un desplazamiento interdepartamental dentro de Colombia o hacia otros países de la región con relación a los datos de ingreso y hospedaje suministrados en frontera a la UAEMC por parte de los inmigrantes venezolanos, la cantidad efectivamente radicada en estos departamentos representa una proporción muy importante de la PEA.

\footnotetext{
${ }^{6}$ A partir del Decreto 542 del 21 de marzo del año 2018 se empezó a realizar el Registro Administrativo de Migrantes Venezolanos en Colombia (RAMV), el cual constó de una operación de registro que se realizó entre el 6 de abril hasta el 8 de junio del 2018, por parte de diferentes organizaciones bajo la coordinación de la Unidad Nacional para la Gestión del Riesgo de Desastres (UNGRD). El RAMV se llevó a cabo en 1.019 puntos habilitados en 30 departamentos y 413 municipios del país.

7 Considerando la información suministrada por el RAMV, aproximadamente el 95\% de los venezolanos censados ingresaron al país en un año posterior al 2016 inclusive.
} 


\section{Gráfico 1. Migración como Porcentaje de la Población Económica Activa 2017 - 2018}

\section{Panel A}

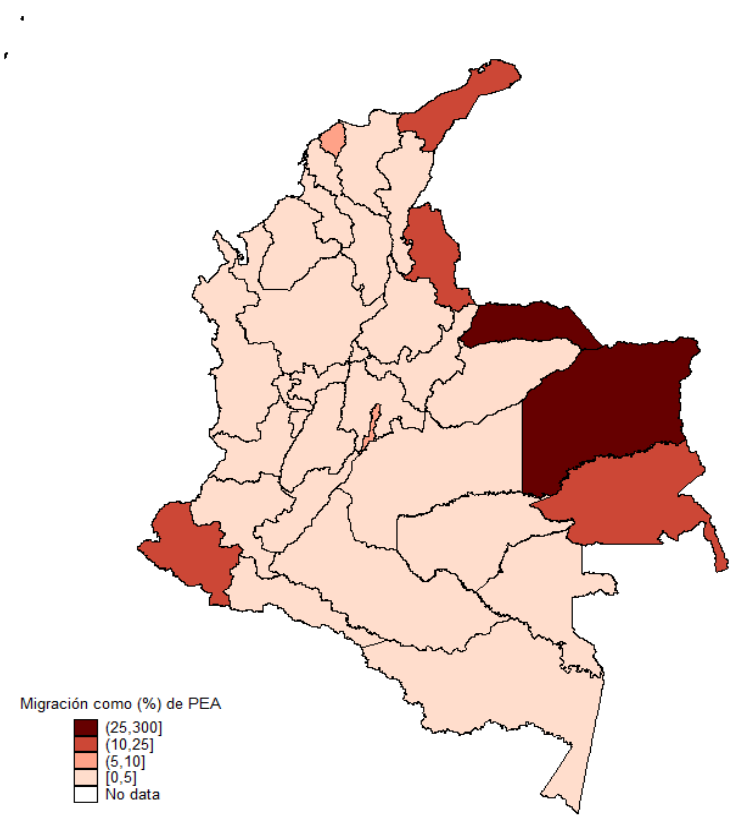

Panel B

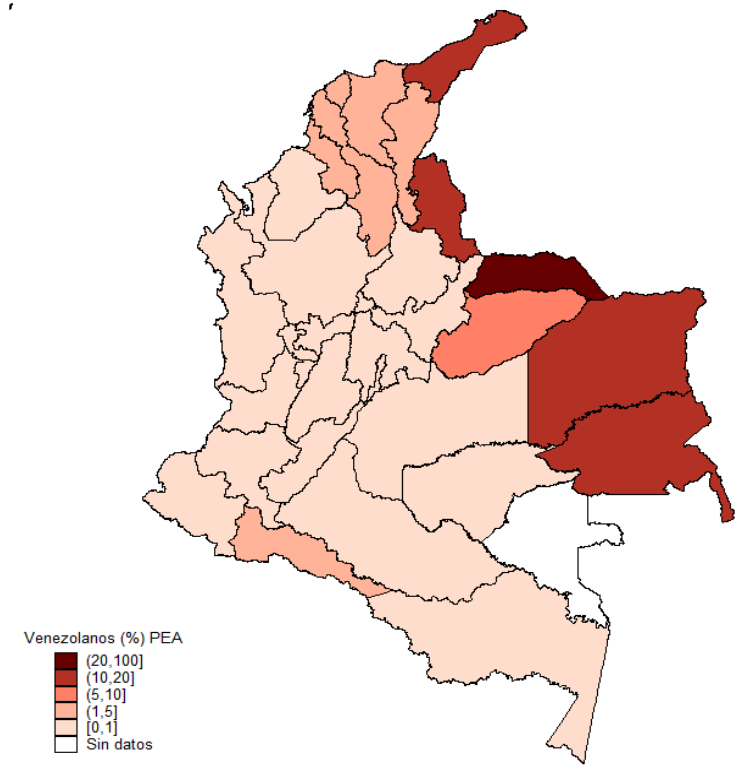

Elaboración propia con base en datos del Departamento Administrativo Nacional de Estadística (DANE), la Unidad Administrativa Especial Migración Colombia (UAEMC) y el Registro Administrativo de Migrantes Venezolanos (RAMV). La población económicamente activa de Amazonas y Casanare corresponden a la del 2015. Para el resto de los departamentos se consideró la PEA del año 2016. El Panel A considera el ratio entre el ingreso migratorio reportado en UAEMC y la PEA de cada departamento, el Panel B muestra el ratio de los venezolanos radicados y censados en el RAMV y la PEA de cada departamento.

Por su parte, Nariño es un departamento no fronterizo en el cual el flujo migratorio fue significativamente alto con relación a la PEA (6.45\%), sin embargo, tal y como se observa en el Panel B del Gráfico 1 y en la tercera columna de la Tabla A1, este departamento en realidad parecería haber funcionado como región de tránsito más que de radicación de los migrantes venezolanos.

En el resto de los departamentos de Colombia el ingreso migratorio fue en la mayoría de los casos de un valor inferior a 1 punto porcentual. Dada la disponibilidad de datos de la Encuesta de Hogares de los departamentos de Colombia proporcionados por el DANE, en este trabajo se enfocará el análisis en los departamentos de La Guajira y de Norte de Santander.

Si se analiza la información migratoria en el tiempo se puede observar que a partir de la apertura de las fronteras entre los dos países - segundo semestre del 2016 - y debido a la crisis económica venezolana, el ingreso migratorio se intensificó en los semestres siguientes (Ver Gráfico 2). A nivel nacional el flujo migratorio acumulado pasó de ser de 150.000 personas semestralmente a un poco más de 700.000 personas en el primer semestre del año 2018.

Por su parte, en los departamentos de La Guajira y Norte de Santander el ingreso migratorio también siguió el mismo patrón presentado a nivel nacional incrementándose la cantidad de migrantes que ingresaron a esos departamentos semestralmente a partir de la apertura de fronteras. 


\section{Gráfico 2. Evolución de los Ingresos Migratorios de Venezolanos por Departamento de Hospedaje. 2012-2018}

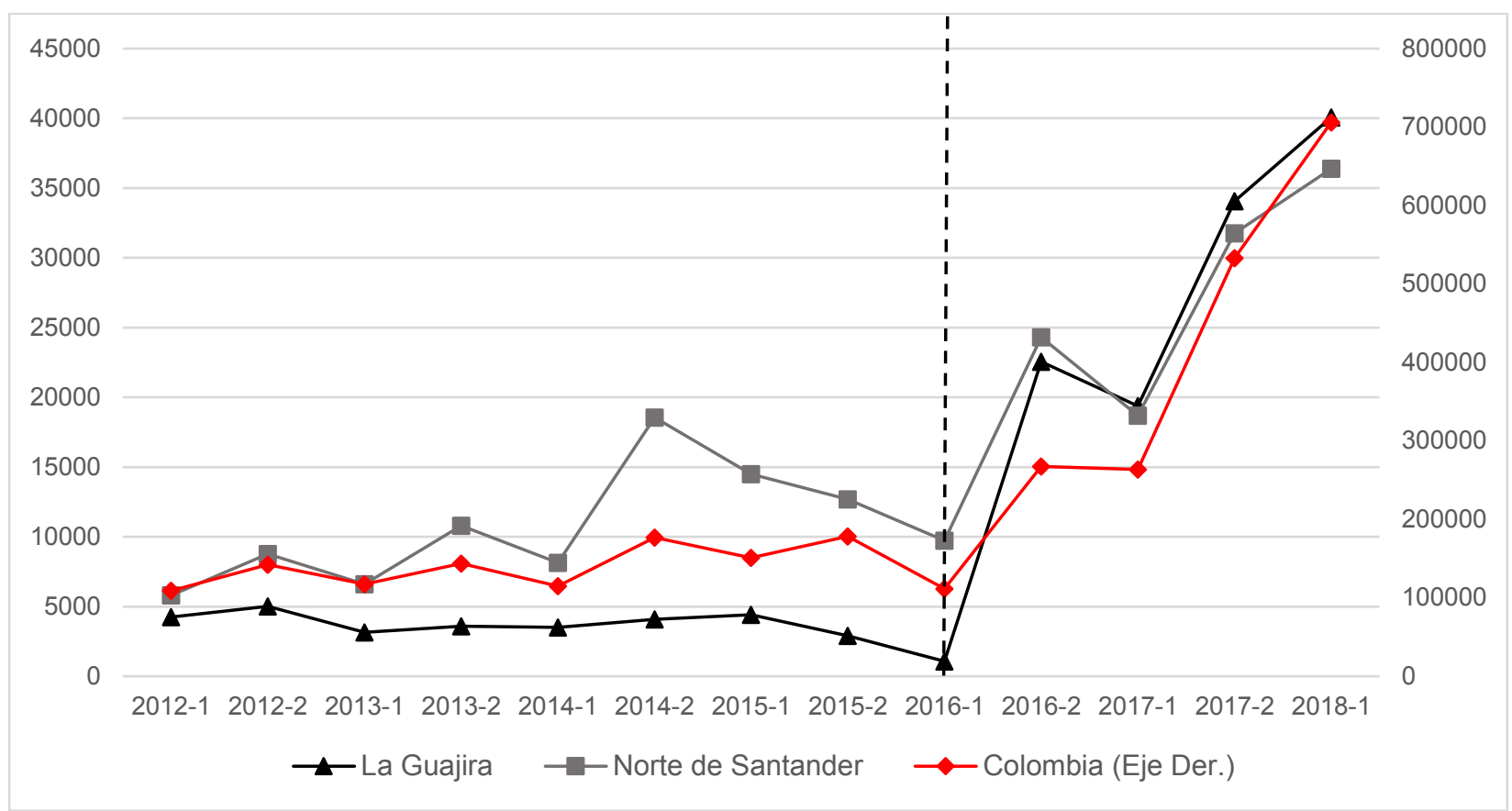

Fuente: Elaboración propia con base en datos de la Unidad Administrativa Especial Migración Colombia (UAEMC).

Si se consideran los ingresos hasta el segundo semestre del año 2017 (Ver Tabla A1 del Apéndice) se puede apreciar que, para el caso de La Guajira y Norte de Santander, la participación de la migración en proporción a la PEA de los departamentos es de $15.67 \%$ y $11.66 \%$, respectivamente; es decir, se puede observar que el ingreso migratorio es muy alto post apertura de las fronteras y aumenta en los semestres siguientes.

\section{Características de los migrantes venezolanos}

Analizando las heterogeneidades en términos de sexo y de edad de los flujos migratorios se puede apreciar en la Tabla 1 presentada a continuación que, de acuerdo con los datos de Migración Colombia, para los dos departamentos bajo estudio, la proporción de hombres y mujeres de Venezuela que migraron a esas regiones entre el 2016 y el 2017 es muy similar. Se observa una mayor proporción para el caso de los hombres pero con una diferencia que no resultaría muy relevante en términos de los efectos que puedan tener sobre el mercado de trabajo.

Esta paridad se presenta también si se consideran los datos de venezolanos radicados irregularmente a nivel nacional en el panel inferior de la Tabla 1 según los datos del RAMV. En términos educativos, por su parte, el $20.15 \%$ de la población encuestada en el RAMV a nivel nacional posee Básica Primaria, el $56.07 \%$ Básica Secundaria, mientras que el $17.44 \%$ posee educación superior (Universitaria, Técnica o Tecnóloga) y tan solo el $0.39 \%$ posee un posgrado.

Adicionalmente, de los datos reportados por el RAMV se puede observar que aproximadamente el $63.72 \%$ de la población venezolana censada a nivel nacional se encuentra en situación de informalidad laboral, desempleo o trabaja independientemente, mientras que tan solo el $0.75 \%$ se encuentra en un empleo formal. Esta información indicaría que debería haber un mayor efecto del aumento en la oferta laboral sobre las variables del mercado de trabajo de la población de 
informales de los departamentos de La Guajira y el Norte de Santander, en particular, no así con la población de ocupados formales.

\section{Tabla 1. Estadísticas Descriptivas}

Flujo Migratorio - Ingresos

\begin{tabular}{|c|c|c|c|}
\hline \multicolumn{2}{|c|}{ Categoría } & La Guajira & $\begin{array}{c}\text { Norte de } \\
\text { Santander }\end{array}$ \\
\hline \multirow{2}{*}{ Sexo } & Femenino & $47.99 \%$ & $45.76 \%$ \\
\hline & Masculino & $52.01 \%$ & $54.24 \%$ \\
\hline \multirow{3}{*}{ Edad } & $18-29$ & $40.02 \%$ & $41.34 \%$ \\
\hline & $30-49$ & $49.26 \%$ & $44.73 \%$ \\
\hline & $50-69$ & $10.72 \%$ & $13.93 \%$ \\
\hline \multicolumn{4}{|c|}{ A nivel nacional - RAMV } \\
\hline \multirow{2}{*}{ Sexo } & Femenino & \multicolumn{2}{|c|}{$49.67 \%$} \\
\hline & Masculino & \multicolumn{2}{|c|}{$50.24 \%$} \\
\hline \multirow{3}{*}{ Edad } & $0-11$ & \multicolumn{2}{|c|}{$20.02 \%$} \\
\hline & $12-17$ & \multicolumn{2}{|c|}{$6.81 \%$} \\
\hline & 18 o más & \multicolumn{2}{|c|}{$73.17 \%$} \\
\hline \multirow{6}{*}{ Educación } & Básica Primaria & \multicolumn{2}{|c|}{$20.15 \%$} \\
\hline & Básica Secundaria & \multicolumn{2}{|c|}{$56.07 \%$} \\
\hline & Técnico - Tecnólogo & \multicolumn{2}{|c|}{$6.76 \%$} \\
\hline & Universitario & \multicolumn{2}{|c|}{$10.67 \%$} \\
\hline & Posgrado & \multicolumn{2}{|c|}{$0.39 \%$} \\
\hline & No Aplica & \multicolumn{2}{|c|}{$5.97 \%$} \\
\hline & $\begin{array}{l}\text { Empleo Informal } \\
\text { Trabajador }\end{array}$ & \multicolumn{2}{|c|}{$23.64 \%$} \\
\hline & Independiente & \multicolumn{2}{|c|}{$21.89 \%$} \\
\hline Tipo de & Desempleado & \multicolumn{2}{|c|}{$18.19 \%$} \\
\hline Actividad & Hogar & \multicolumn{2}{|c|}{$9.72 \%$} \\
\hline & Estudiante & \multicolumn{2}{|c|}{$7.72 \%$} \\
\hline & Empleado Formal & \multicolumn{2}{|c|}{$0.75 \%$} \\
\hline & No Reporta & \multicolumn{2}{|c|}{$18.09 \%$} \\
\hline
\end{tabular}

Notas. Fuente: Elaboración propia con base en datos de la Unidad Administrativa Especial Migración Colombia (UAEMC) y del Registro Administrativo de Migrantes Venezolanos en Colombia (RAMV).

Dada la información presentada tanto por parte de la UAEMC como del RAMV, los datos son concluyentes y permiten verificar que, efectivamente, post apertura de las fronteras entre Colombia y Venezuela por parte de Nicolás Maduro en el segundo semestre del año 2016 ha habido un flujo fenomenal de migrantes venezolanos hacia Colombia que, principalmente, se radicaron en zonas fronterizas entre los dos países.

A su vez, la información suministrada sugiere que el flujo migratorio efectivamente se ha traducido en un incremento importante en la oferta laboral de La Guajira y Norte de Santander, lo cual representa un shock exógeno en el mercado laboral colombiano que potencialmente podría haber afectado el salario real. En las siguientes secciones, por lo tanto, se propondrán diferentes 
estrategias que permitan estimar si tal efecto realmente se dio sobre los salarios con base en la información presentada hasta el momento.

\section{EFECTO DE LA MIGRACIÓN VENEZOLANA SOBRE LOS SALARIOS}

Una primera aproximación para conocer el efecto de la migración sobre el salario real en las regiones fronterizas de Colombia consiste en analizar la evolución en el tiempo de los salarios de La Guajira y Norte de Santander. Esta información se presenta en el Gráfico 3.

Se puede observar que, post apertura de las fronteras e ingreso de migrantes a los departamentos de La Guajira y Norte de Santander, el salario horario real medido en logaritmos cayó fuertemente para esos departamentos, mientras que en el caso del promedio para Colombia la tendencia no fue igual. De hecho, en ese periodo se presentó un leve incremento para el promedio colombiano, lo cual permite visualizar que parecería haber un efecto negativo sobre los salarios debido al incremento en la oferta de trabajo.

Gráfico 3. Evolución del Salario Real Horario. 2014-2018

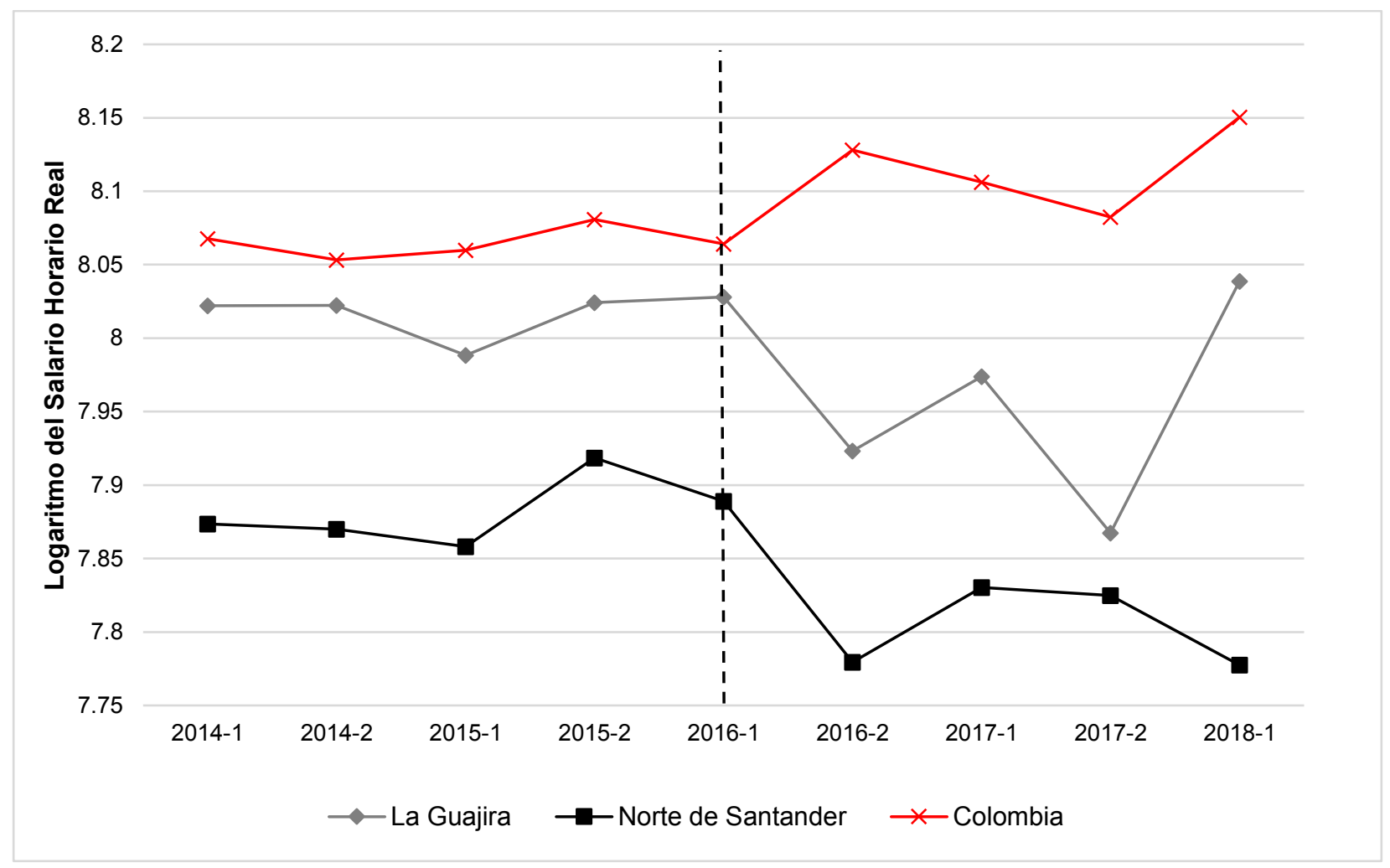

Notas. Fuente: Elaboración propia con base en datos del Departamento Administrativo Nacional de Estadística (DANE).

Sin embargo, dado que pueden existir múltiples problemas de interpretación causal al comparar las regiones afectadas y el resto del país mediante un promedio generalizado del salario real, en las siguientes subsecciones se implementarán metodologías alternativas que permitan corroborar el impacto causal de la migración sobre los salarios reales en estas regiones colombianas. 


\section{Identificación y método de estimación}

En primer lugar es importante poder descomponer el efecto de la migración sobre los salarios en dos: por un lado, el efecto específico del incremento en el flujo migratorio con el respectivo mecanismo causal especificado en las secciones anteriores y, por otro lado, despojar a la variación del salario real del componente que se encuentra explicado por elementos macroeconómicos que afectaron a todas las regiones conjuntamente.

El escenario ideal para el análisis es uno en el cual se cuente con un contrafactual de las unidades afectadas por el flujo migratorio que permitan comparar cómo hubiera sido la evolución del salario real en ausencia del shock de oferta laboral y, de esta manera, mirar la diferencia con el sendero del salario real efectivamente ocurrido en el tiempo. Sin embargo, dada la imposibilidad práctica de contar con un contrafactual, en la literatura se encuentran distintas metodologías que permiten aproximar el análisis para determinar el impacto causal del shock bajo estudio.

La metodología más recurrente en la literatura económica consiste en comparar a las regiones o unidades afectadas por el shock exógeno (Grupo Tratamiento) con un conjunto de unidades que funcionen como control (Grupo Control) las cuales deben cumplir con ciertos requisitos para permitir la comparabilidad entre los distintos grupos bajo análisis.

El supuesto fundamental que se debe cumplir con relación al grupo de control para poder determinar el impacto causal sobre el grupo de tratamiento del shock migratorio consiste en que, en ausencia del shock, la tendencia en los salarios reales de los departamentos del grupo de tratamiento y del grupo de control sería igual. Dado que no se cuenta con el contrafactual postshock migratorio se debe asegurar entonces que la tendencia de la variable de interés para ambos grupos, pre-shock migratorio, sean similares.

Surge entonces un problema no menor y consiste en determinar la composición o la forma según la cual se construiría ese grupo de control que permita comparar la evolución de los salarios reales de La Guajira y de Norte de Santander.

En este trabajo se exploran dos alternativas que pueden considerase como un complemento para agregar robustez a los resultados obtenidos. Por un lado, se estima el impacto causal del flujo migratorio sobre el salario real doméstico en los grupos de tratamiento mediante la metodología de diferencias en diferencias tomando como grupo de control a los departamentos de Antioquia, Caquetá y el Chocó. Es decir, en una primera instancia se realizarán las estimaciones basando el análisis en una selección de un grupo de control ad-hoc, seleccionando aquellas regiones cuyas tendencias en términos del salario sean lo más parecido posible a la del grupo de tratamiento.

Adicionalmente, en una segunda instancia, las estimaciones se realizarán construyendo un grupo de control mediante la Metodología de Control Sintético siguiendo lo desarrollado por Abadie y Gardeazabal (2003) y Abadie, Diamond y Hainmueller (2010). Bajo esta estrategia, se construye un grupo de control a partir del resto de los departamentos de Colombia que no se vieron afectados por el flujo migratorio significativamente, otorgándoles diferentes niveles de ponderación, mediante un análisis estadístico que se detallará más adelante.

Se mostrará en los resultados que, independientemente de la metodología implementada para la construcción del grupo de control, la estimación es robusta y el efecto causal estimado del incremento en la oferta laboral sobre el salario real doméstico es muy similar para las dos metodologías propuestas. 


\section{Grupo de Control Ad-Hoc}

Como se expuso previamente en esta subsección se empleará la metodología de diferencias en diferencias para estimar el impacto causal del incremento en la oferta laboral debido al aumento en la migración desde Venezuela.

Dado el importante flujo de migración de venezolanos que recibieron los departamentos de La Guajira y Norte de Santander y debido a la disponibilidad de datos para la medición del salario horario promedio de esas regiones, estos dos departamentos serán considerados como grupos de tratamiento. Por su parte, bajo esta metodología el grupo de control se construirá ad-hoc para lo cual se seleccionaron a los departamentos de Antioquia, Caquetá y Chocó.

La selección de estos departamentos se basó principalmente en que son regiones no fronterizas, con lo cual se espera que la apertura de las fronteras entre Colombia y Venezuela y el posterior flujo migratorio no haya afectado la tendencia de los salarios de manera significativa. Adicionalmente porque, tal y como se presentó en la Tabla A1, la migración acumulada para esas regiones con relación a la PEA fue inferior a los 2 puntos porcentuales y, de acuerdo con el RAMV, los venezolanos radicados representan menos de 1 punto porcentual de la PEA.

Por último, de acuerdo con lo que se observa en el Gráfico 4, el comportamiento tendencial en el salario real horario del grupo de tratamiento y de control propuestos medido en logaritmos es muy similar pre-apertura de las fronteras entre Colombia y Venezuela. Sin embargo, a partir del segundo semestre del año 2016, luego de la línea punteada, el salario real horario de los departamentos del grupo de tratamiento cae fuertemente y adquiere una tendencia muy distinta a la del grupo de control, evidencia que podría respaldar inicialmente que habría un efecto negativo del incremento en la oferta laboral sobre el salario real horario.

Gráfico 4. Evolución del Salario Real Horario. 2014-2018

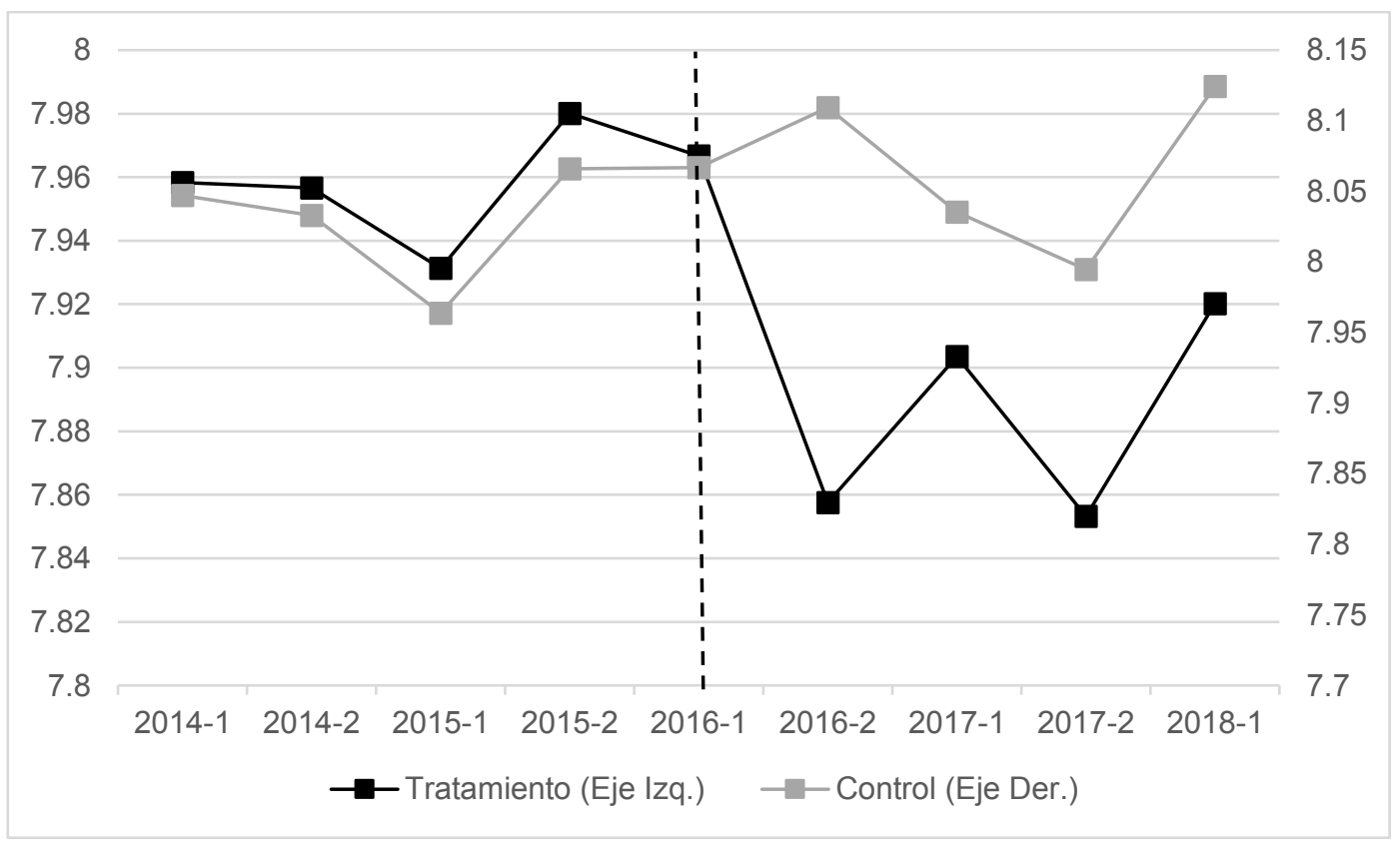

Notas. Fuente: Elaboración propia con base en datos del Departamento Administrativo Nacional de Estadística (DANE). En el Grupo de Tratamiento se consideraron a los departamentos de La Guajira y Norte de Santander; en el Grupo de Control se consideraron a los departamentos de Antioquia, Chocó y Caquetá. 
Para verificar que la tendencia del salario real horario en logaritmos entre el grupo de tratamiento y el grupo de control es igual en los periodos pre-tratamiento, se presenta en la Tabla A2 del Anexo el estadístico $\mathrm{F}$ y el $\mathrm{P}$-Valor del correspondiente test de tendencia común pre-tratamiento entre el grupo de control ad-hoc y el grupo de tratamiento.

De acuerdo con lo que se observa en la Tabla A2, no se puede rechazar la hipótesis nula de igual tendencia pre-tratamiento en la variable de interés lo cual sugiere que la utilización del grupo de control ad-hoc conformado por Antioquia, Caquetá y el Chocó es adecuada.

Para poder estimar el efecto del incremento en la oferta laboral sobre el salario real de los individuos que habitan en los departamentos del grupo de tratamiento, se implementa la metodología de diferencias en diferencias. Se realizan estimaciones econométricas que permitan incorporar controles a nivel individual y agregados por departamento y periodo para así, de esta manera, evitar sesgos en las estimaciones obtenidas debido a la omisión de variables relevantes.

El modelo econométrico que se pretende estimar para poder cumplir con el objetivo es el siguiente:

$$
\log (w)_{i d t}=\alpha+\delta X_{i d t}+\theta Z_{i d t}+\pi_{d}+\sigma_{t}+\mu_{i d t}
$$

La variable $w_{i d t}$ denota el salario real horario de la observación $i$, la variable $X_{i d t}$ toma valor igual a 1 si la observación i pertenece al Grupo de Tratamiento y pertenece al periodo post apertura de fronteras y toma valor 0 en caso contrario. Las variables $\pi_{D} y \sigma_{t}$ son efectos fijos por departamento y por periodo, respectivamente $\mathrm{y}$, por último, el vector $Z_{i d t}$ controla por las heterogeneidades de los individuos considerados para el estudio que podrían sesgar los resultados de las estimaciones realizadas. El coeficiente de interés que reflejaría el efecto causal sobre el salario del flujo migratorio mediante la metodología de diferencias en diferencias es $\delta$. Los resultados de las estimaciones se presentan a continuación en la Tabla 2.

Tabla 2. Estimaciones Agregadas.

Variable Dependiente: Logaritmo del Salario Horario Real

\begin{tabular}{lcc}
\hline & \multicolumn{2}{c}{ Control Ad-Hoc } \\
\hline & $(1)$ & $(2)$ \\
\hline & & \\
Efecto Migración & $\mathbf{0 . 1 0 0 * * *}$ & $-\mathbf{0 . 0 9 6 6 * * *}$ \\
& $\mathbf{( 0 . 0 1 5 2 )}$ & $\mathbf{( 0 . 0 1 5 3 )}$ \\
Observaciones & 35,576 & 35,576 \\
$\mathrm{R}^{2}$ & 0.330 & 0.362 \\
\hline Controles Individuales & Sí & Sí \\
Controles Agregados y Temporales & No & Sí \\
\hline Notas. Errores estándar robustos y clusterizados a nivel periodo-departamento entre paréntesis. Las \\
estrellas ${ }^{*},{ }^{* *}$ y** denotan significatividad al 10, 5 y 1 por ciento. Las observaciones corresponden al \\
periodo 2014-2018 con datos de junio y diciembre de cada año hasta la primera mitad del 2018. Los \\
controles individuales incluyen características vinculadas con el sexo del individuo, su nivel de \\
educación lineal y al cuadrado, la edad lineal y al cuadrado, si es un trabajador formal o no y el estado \\
civil. Los controles agregados y temporales se vinculan con dummies regionales, temporales y de rama \\
de actividad del individuo. Fuente: Elaboración propia con base en la GEIH.
\end{tabular}

De las estimaciones presentadas se observa que, luego de controlar por características individuales y por elementos temporales y regionales, el efecto estimado causal del incremento 
en la oferta laboral debido al flujo migratorio post apertura de las fronteras entre Colombia y Venezuela, sobre el salario horario real de los individuos de La Guajira y Norte de Santander, es un efecto negativo y estadísticamente significativo para los niveles habituales de significatividad. En la columna 2 de la Tabla 2 se observa que el efecto sería de una caída en promedio de aproximadamente $9.7 \%$ del salario real horario, ceteris paribus.

\section{Método de Control Sintético}

La otra metodología propuesta en esta parte del trabajo podría solucionar los problemas de discrecionalidad que se le pueden atribuir a las estimaciones mediante una selección de un grupo de control ad-hoc tal y como se hizo en la subsección anterior.

La intuición del Método de Control Sintético se basa en determinar una combinación lineal óptima de las unidades de control -en este análisis los departamentos que no se vieron fuertemente afectados por el flujo migratorio - de manera tal que, de acuerdo a una ponderación a determinar, se construya una unidad de control sintética que sirva de contrafactual y, consecuentemente, permita realizar las comparaciones entre las unidades de tratamiento o de análisis (La Guajira y Norte de Santander) y la unidad de control construida sintéticamente.

La clave en esta metodología está en determinar la ponderación óptima que se le atribuye a cada uno de los departamentos no afectados por la migración para poder realizar la combinación lineal que permita obtener la unidad de comparación.

Siguiendo a Gardeazabal (2010) y a Abadie, Diamond y Hainmueller (2010) el problema analíticamente a resolver se basa en seleccionar un vector $W^{*}$ de ponderadores que permita minimizar la expresión dada por

$$
W^{*}=\underset{W}{\operatorname{argmin}}\left(X_{1}-X_{0} W\right)^{\prime} V\left(X_{1}-X_{0} W\right)
$$

Sujeto a que:

$$
\begin{gathered}
w_{j} \geq 0(j=1,2, \ldots J) \\
w_{1}+w_{2}+\cdots+w_{J}=1
\end{gathered}
$$

Donde $J$ denota el número de unidades de control disponibles, en este análisis los departamentos de control que no se vieron fuertemente afectados por el flujo migratorio. $W=\left(w_{1}, \ldots, w_{J}\right)$ es un vector no negativo de ponderadores para cada una de las unidades de control disponibles que debe sumar 1 de acuerdo a las restricciones previamente expuestas. $X_{1}$ es un vector de dimensiones $(K \times 1)$ con valores de $\mathrm{K}$ características relevantes para la unidad tratada y $X_{0}$ es una matriz de dimensión $(K \times J)$ que contiene los mismos valores para las mismas $\mathrm{K}$ variables, pero para las $\mathrm{J}$ unidades de control bajo análisis.

Por último, $V$ es una matriz diagonal con componentes no-negativos en la cual se explicita la importancia relativa de cada una de las características seleccionadas como determinantes de la variable de interés bajo análisis, en este caso el salario horario real.

Luego, una vez minimizada la expresión previamente presentada, sujeta a las restricciones de no negatividad y de suma igual a la unidad, se determinaría el vector $W^{*}$ de ponderaciones óptimas el cual, si se multiplica por la matriz $X_{0}$, permitirá encontrar los valores de las variables especificadas ponderadas, esto es pertenecientes a la unidad contrafactual de comparación.

Por último, definiendo $Y_{1}$ como un vector de dimensión $(T \times 1)$ que contiene los valores de la variable de interés, en este caso el salario real horario y definiendo a $Y_{0}$ como una matriz de 
$(T \times J)$ con las mismas características que $Y_{1}$ solamente que para todas las unidades de control consideradas, se puede determinar el valor de la variable de interés sintética, esto es el salario horario real sintético a partir de los ponderadores obtenidos y la matriz $Y_{0}$.

Con lo cual, el vector de salarios horarios reales sintéticos estaría dado por $Y_{1}^{*}=Y_{0} W^{*}$, por lo tanto comparando $Y_{1}$, que es el valor real del salario horario en términos reales, con $Y_{1}^{*}$, que es el valor sintético encontrado que tomaría el rol de contrafactual, se podría determinar el efecto causal del incremento en la oferta laboral sobre el salario percibido por los habitantes de La Guajira y Norte de Santander.

Para poder estimar las ponderaciones fue necesario especificar un conjunto de variables que se consideran relevantes para explicar el salario horario real. Una de las limitaciones de la metodología de Control Sintético es que las unidades de análisis son, en general, unidades a nivel agregado, motivo por el cual es importante determinar variables explicativas que permitan explicar el nivel salarial de los departamentos a nivel agregado y no a nivel de cada uno de los individuos.

Dado esto, se seleccionaron las siguientes variables: la proporción de trabajadores en diferentes grandes ramas de la economía, la tasa de desempleo, la tasa de informalidad laboral, los años de educación promedio de cada una de las regiones, la proporción de individuos calificados en cada departamento y el salario horario real promedio en algunos periodos pre-tratamiento específicos. Las ponderaciones correspondientes al vector $W^{*}$ determinadas bajo esta metodología se presentan en la Tabla A3 del Apéndice.

De las tres regiones elegidas ad-hoc en la metodología de diferencias en diferencias presentadas en la sección anterior, el cómputo de $W^{*}$ le otorgó ponderación no nula a una, esto es a Chocó con una ponderación del $17.7 \%$. A su vez, por otro lado, se observa que se incluyeron otros departamentos que no fueron considerados en el análisis en diferencias en diferencias tales como Magdalena, Nariño y Tolima con ponderaciones de $27.5 \%, 5.7 \%$ y $49.1 \%$, respectivamente.

Una vez calculadas las ponderaciones óptimas y computando el valor de la variable de interés sintética se observan los resultados en el Gráfico 5.

De las estimaciones realizadas se obtiene que pre-tratamiento habría una tendencia y valores muy similares entre la unidad de tratamiento ${ }^{8}$ y la de control sintético, sin embargo, post apertura de las fronteras entre Colombia y Venezuela, el salario horario real de la unidad de tratamiento cae mucho más fuertemente mientras que el salario horario real de la unidad de control construida mediante MCS no cae a tal velocidad, manteniéndose una brecha post-tratamiento entre los valores de tratamiento y de control que no existía en los periodos previos a la apertura de las fronteras.

\footnotetext{
${ }^{8}$ Para este análisis y dado que la metodología de Control Sintético permite únicamente considerar una sola unidad como tratamiento, se consideró entonces a La Guajira y Norte de Santander como una sola región estimando el salario horario real promedio para la población de los dos departamentos como si fueran el mismo.
} 


\section{Gráfico 5. Evolución del Salario Real Horario. Grupo de Tratamiento y Grupo de Control Sintético. 2014-2018}

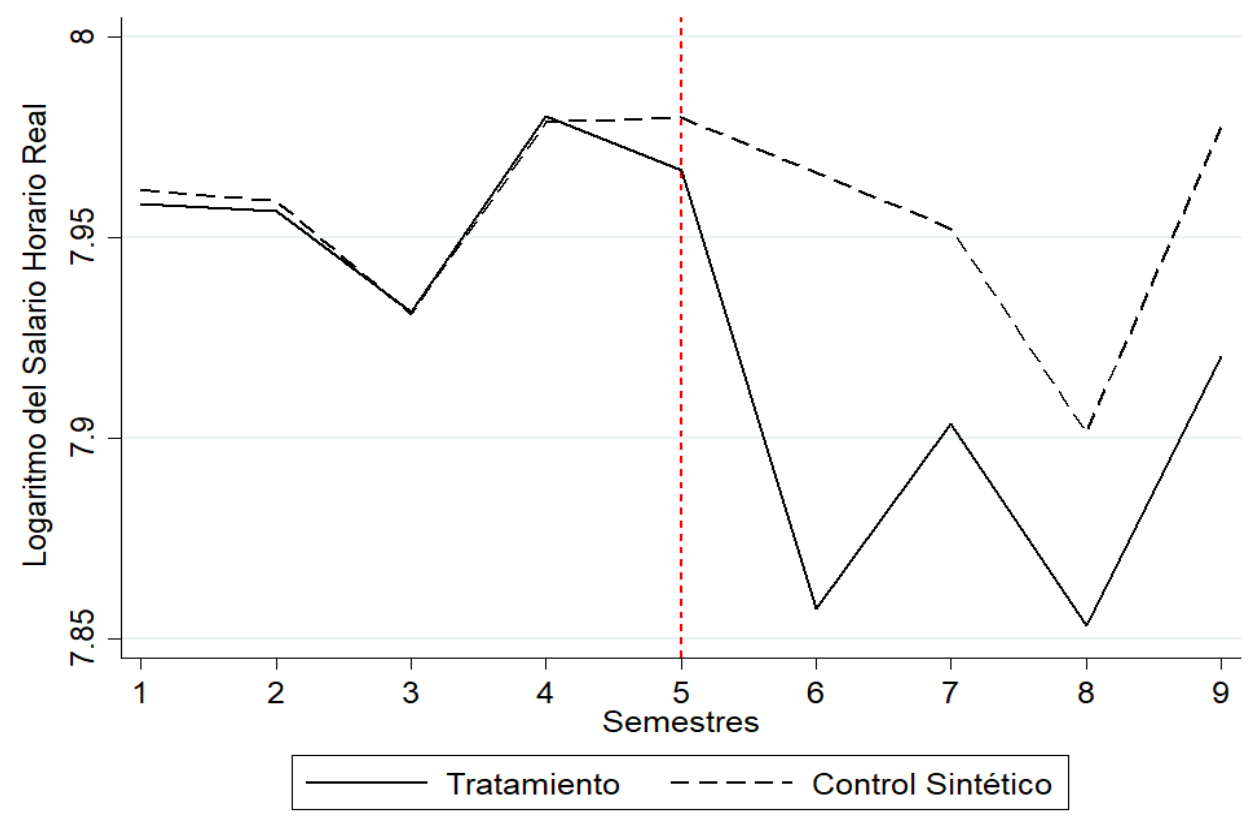

Notas. Fuente: Elaboración propia con base en datos del Departamento Administrativo Nacional de Estadística (DANE). En el Grupo de Tratamiento se consideraron a los departamentos de La Guajira y Norte de Santander.

Si se calcula la diferencia entre el salario promedio pre-tratamiento y post-tratamiento para ambos grupos presentados y, posteriormente, se computa la diferencia de esas diferencias entre los dos grupos se obtiene que el efecto causal estimado promedio fue de una caída del salario real horario para el grupo de tratamiento de $6.2 \%$ aproximadamente, resultado que estaría muy vinculado con el obtenido en las estimaciones agregadas de diferencias en diferencias de la Tabla 2.

\section{EFECTOS HETEROGÉNEOS SOBRE LOS SALARIOS}

En esta parte del trabajo se presentan las estimaciones del efecto tratamiento sobre el salario real horario, pero desagregando por diferentes características de la población bajo análisis. Las variables con base en los cuales se estimará la heterogeneidad en los efectos causales del incremento de la oferta laboral sobre el salario real se basarán en características de nivel de calificación, sexo y formalidad laboral de las personas en los departamentos de La Guajira y Norte de Santander.

Para tal fin, se procederá a incluir interacciones del efecto tratamiento con las características de heterogeneidad que se propone analizar de manera tal de poder determinar si el efecto tratamiento presenta una diferencia estadísticamente significativa entre los subgrupos analizados. La ecuación a estimar para cada uno de los efectos es la siguiente:

$$
\log (w)_{i d t}=\alpha+\delta X_{i d t}+\left(\sum_{d \in D} \tau_{j} \pi_{d}\right) H_{i d t}+\left(\sum_{t \in T} \lambda_{j} \sigma_{t}\right) H_{i d t}+\psi X_{i d t} H_{i d t}+\theta Z_{i d t}+\pi_{d}+\sigma_{t}+\mu_{i d t}
$$

Donde $H$ es una variable que toma valor 1 para alguno de los subgrupos en los cuales se desea ver si el efecto de la migración sobre los salarios fue heterogéneo y 0 (subgrupo base) en caso contrario. En el caso del nivel de calificación $H$ es igual a 1 si el individuo es calificado ( 0 en caso contrario), para analizar la variación salarial por sexo $H$ es igual a 1 para las mujeres ( 0 en caso 
contrario) y, por último, por formalidad laboral $H$ es igual a 1 para los individuos formalmente contratados ( 0 en caso contrario).

Por su parte, el tercer componente de la regresión son las interacciones entre los efectos fijos por departamento y la variable de subgrupos lo cual permite controlar por diferenciales constantes en el tiempo de los subgrupos, pero distintos entre regiones. Mientras que, el cuarto elemento de la ecuación captura diferenciales variables en el tiempo entre los subgrupos analizados pero constantes entre departamentos. El resto de las variables son iguales a las especificadas en la ecuación (1).

El coeficiente estimado $\delta$ indicaría el efecto del incremento en la oferta laboral sobre los salarios del subgrupo base mientras que, el coeficiente $\psi$, indicaría la diferencia en el efecto de la migración sobre los salarios entre uno de los subgrupos mencionado previamente y el correspondiente subgrupo base.

\section{Efecto por Nivel de Calificación}

Para estimar el efecto desagregado por nivel de calificación se dividió a la población en dos grupos: calificados y no calificados. Se definieron a los no calificados como aquellos individuos que tenían un nivel educativo inferior a la secundaria completa y como mano de obra calificada a los individuos con secundaria completa o más.

Con relación al efecto esperado de la migración sobre los salarios por nivel de calificación, de acuerdo con la literatura, es muy común que en los casos de flujos de migrantes hacia otras regiones las posiciones alcanzadas por los trabajadores migrantes sean, en general, menores a las posiciones de trabajo que poseen los trabajadores residentes con iguales características en términos de educación y experiencia (Dustmann et al. 2016).

Este fenómeno conocido como downgrading implicaría que, independientemente de la composición educativa de los flujos de migrantes de una región a otra, el mayor incremento relativo de la oferta laboral se presentaría en aquellos empleos de menor calificación, con lo cual, ceteris paribus, el salario real de esos tipos de empleos debería caer mucho más en términos relativos. (Para ver evidencia de downgrading ver por ejemplo Borjas, 1985; Dustmann y Preston, 2012; Dustmann, Frattini y Preston, 2013)

De acuerdo con lo estimado se observa en la Tabla 3 que la caída en el salario real fue mucho más fuerte en el caso de los trabajadores no calificados en comparación a los calificados. Los empleados de baja calificación sufrieron una caída del salario real, en promedio, 7 puntos porcentuales mayor en comparación a los trabajadores calificados, diferencia que representa, aproximadamente, una caída en el salario real de casi el doble para los no calificados en comparación a los calificados. 
Tabla 3. Efectos Heterogéneos por Calificación

Variable Dependiente: Logaritmo del Salario Horario Real

\begin{tabular}{lcc}
\hline & \multicolumn{2}{c}{ Control Ad-Hoc } \\
\hline & $(1)$ & $(2)$ \\
Efecto Migración & $-\mathbf{0 . 1 5 0 * * *}$ & $-\mathbf{0 . 1 4 1 * * *}$ \\
& $(\mathbf{0 . 0 2 4 5 )}$ & $\mathbf{( 0 . 0 2 4 5 )}$ \\
Efecto Migración $\times$ Calificado & $\mathbf{0 . 0 7 8 6 * * *}$ & $\mathbf{0 . 0 7 1 4 * *}$ \\
& $\mathbf{( 0 . 0 2 8 2 )}$ & $\mathbf{( 0 . 0 2 7 5 )}$ \\
Observaciones & 35,576 & 35,576 \\
$\mathrm{R}^{2}$ & 0.335 & 0.365 \\
\hline Controles Individuales & Sí & Sí \\
Controles Agregados y Temporales & No & Sí \\
\hline
\end{tabular}

Notas. Errores estándar robustos y clusterizados a nivel periodo-departamento entre paréntesis. Las estrellas *, ${ }^{* *} y^{* * *}$ denotan significatividad al 10,5 y 1 por ciento. Las observaciones corresponden al periodo 2014-2018 con datos de junio y diciembre de cada año hasta la primera mitad del 2018. Los controles individuales incluyen características vinculadas con el sexo del individuo, su nivel de educación lineal y al cuadrado, la edad lineal y al cuadrado, si es un trabajador formal o no y el estado civil. Los controles agregados y temporales se vinculan con dummies regionales, temporales y de rama de actividad del individuo. Fuente: Elaboración propia con base en la GEIH.

Los resultados presentados en la Tabla 3 son consistentes con la evidencia a favor de la existencia de downgrading. Esto sugiere por lo tanto que, pese a que los migrantes puedan tener altos niveles de calificación, se estarían ubicando en empleos de baja calificación generando una presión sobre el salario real en estos segmentos del mercado de trabajo. ${ }^{9}$

\section{Efecto por Sexo}

Las estimaciones del efecto tratamiento por sexo se hicieron siguiendo la misma metodología presentada previamente de diferencias en diferencias basando el análisis en el mismo grupo control ad-hoc propuesto con anterioridad. Los resultados se presentan en la Tabla 4 a continuación:

\footnotetext{
${ }^{9}$ Este análisis sin embargo es válido principalmente en el corto plazo, dado que como Dustmann et al (2016) sugieren, con el paso del tiempo se presentaría un fenómeno contrario de upgrading en el cual los trabajadores inmigrantes habrían adquirido la experiencia suficiente como para participar como mano de obra en trabajos de mejor calificación o más relacionados con sus características educativas y de experiencia profesional.
} 
Tabla 4. Efectos Heterogéneos por Sexo

Variable Dependiente: Logaritmo del Salario Horario Real

\begin{tabular}{|c|c|c|}
\hline & \multicolumn{2}{|c|}{ Control Ad-Hoc } \\
\hline & $(1)$ & $(2)$ \\
\hline \multirow[t]{2}{*}{ Efecto Migración } & $-0.119 * * *$ & $-0.116 * * *$ \\
\hline & (0.0199) & $(0.0190)$ \\
\hline \multirow[t]{2}{*}{ Efecto Migración×Mujer } & 0.0382 & 0.0399* \\
\hline & $(0.0236)$ & $(0.0225)$ \\
\hline Observaciones & 35,576 & 35,576 \\
\hline $\mathrm{R}^{2}$ & 0.331 & 0.363 \\
\hline Controles Individuales & Sí & Sí \\
\hline Controles Agregados y Temporales & No & Sí \\
\hline \multicolumn{3}{|c|}{$\begin{array}{l}\text { Notas. Errores estándar robustos y clusterizados a nivel periodo-departamento entre } \\
\text { paréntesis. Las estrellas }{ }^{*},{ }^{* *} y^{* * *} \text { denotan significatividad al } 10,5 \text { y } 1 \text { por ciento. Las } \\
\text { observaciones corresponden al periodo } 2014-2018 \text { con datos de junio y diciembre de cada } \\
\text { año hasta la primera mitad del } 2018 \text {. Los controles individuales incluyen características } \\
\text { vinculadas con el nivel de educación lineal y al cuadrado, la edad lineal y al cuadrado, si es } \\
\text { un trabajador formal o no y el estado civil. Los controles agregados y temporales se vinculan } \\
\text { con dummies regionales, temporales y de rama de actividad del individuo. Fuente: } \\
\text { Elaboración propia con base en la GEIH. }\end{array}$} \\
\hline
\end{tabular}

Tal y como se evidencia, el efecto tratamiento entre hombres y mujeres, luego de controlar por características observables y no observables, indica una caída más fuerte en el salario horario real para los hombres en aproximadamente 4 puntos porcentuales, diferencia que es estadísticamente significativa a los niveles habituales de significatividad.

La mayor caída para el caso de los hombres podría ser consistente con una asignación de roles tradicional al interior de los hogares, lo cual generaría que la oferta de trabajo de los hombres aumente mucho más en comparación a la de las mujeres produciendo, de esta manera, una caída salarial más importante para los trabajadores de sexo masculino.

\section{Efecto por Informalidad Laboral}

Siguiendo en línea con el análisis de efectos heterogéneos de la caída del salario real post apertura de las fronteras entre Colombia y Venezuela en el segundo semestre del año 2016, en esta sección se estima el efecto tratamiento dividiendo a la población en dos categorías: informales y formales.

Se consideró a los trabajadores como informales si el empleado no cotiza a un fondo de pensiones, si en caso de cotizar a un fondo de pensiones el total del aporte es realizado por el empleado o bien si el empleado no se encuentra como afiliado en un régimen contributivo de salud, es decir una categorización en la cual se pone énfasis en los derechos laborales del empleado.

A partir de esta categorización se estima si hay un efecto diferencial de la migración sobre los salarios entre los dos grupos. Los resultados se presentan en la siguiente tabla: 
Tabla 5. Efectos Heterogéneos por Formalidad Laboral

Variable Dependiente: Logaritmo del Salario Horario Real

\begin{tabular}{|c|c|c|}
\hline & \multicolumn{2}{|c|}{ Control Ad-Hoc } \\
\hline & (1) & $(2)$ \\
\hline \multirow[t]{2}{*}{ Efecto Migración } & $-0.135 * * *$ & $-0.128 * * *$ \\
\hline & $(0.0216)$ & $(0.0214)$ \\
\hline \multirow[t]{2}{*}{ Efecto Migración ×Formal } & $0.0957 * * *$ & $0.0943 * * *$ \\
\hline & $(0.0276)$ & $(0.0254)$ \\
\hline Observaciones & 35,576 & 35,576 \\
\hline $\mathrm{R}^{2}$ & 0.333 & 0.363 \\
\hline Controles Individuales & Sí & Sí \\
\hline Controles Agregados y Temporales & No & Sí \\
\hline \multicolumn{3}{|c|}{$\begin{array}{l}\text { Notas. Errores estándar robustos y clusterizados a nivel periodo-departamento entre paréntesis. } \\
\text { Las estrellas }{ }^{*},{ }^{* \star} \text { y }{ }^{* *} \text { denotan significatividad al } 10,5 \text { y } 1 \text { por ciento. Las observaciones } \\
\text { corresponden al periodo } 2014-2018 \text { con datos de junio y diciembre de cada año hasta la primera } \\
\text { mitad del } 2018 \text {. Los controles individuales incluyen características vinculadas con el sexo del } \\
\text { individuo, su nivel de educación lineal y al cuadrado, la edad lineal y al cuadrado y el estado civil. } \\
\text { Los controles agregados y temporales se vinculan con dummies regionales, temporales y de rama } \\
\text { de actividad del individuo. Fuente: Elaboración propia con base en la GEIH. }\end{array}$} \\
\hline
\end{tabular}

En promedio el salario cayó, aproximadamente, 9 puntos porcentuales más en el caso de los trabajadores informales frente a los formales, siendo esta diferencia estadísticamente significativa. La magnitud y la dirección del coeficiente que se obtiene es consistente con lo que se esperaría considerando que la población de trabajadores informales se encuentra en mayores condiciones de vulnerabilidad ante un incremento en la oferta de trabajo y que, tal y como se mostró con anterioridad, los migrantes venezolanos estarían participando principalmente en el mercado de trabajo informal.

\section{ANÁLISIS DE ROBUSTEZ}

\section{Método de Control Sintético}

Dados los supuestos realizados, con relación a la conformación de los Grupos de Control para estimar el efecto causal del incremento en la oferta laboral debido a un shock migratorio post apertura de las fronteras con Venezuela en el segundo semestre del año 2016, en esta parte del trabajo se presentan diferentes ejercicios de robustez para garantizar que los resultados puedan ser interpretados causalmente.

En primer lugar, en el Gráfico 6 se observa el ejercicio de estimar la evolución de la brecha entre el grupo de tratamiento y su respectivo Grupo de Control Sintético cambiando el grupo de tratamiento y considerando a diferentes regiones de Colombia, distintas a La Guajira y Norte de Santander, como si hubieran sido afectadas por la migración tal y como lo sugieren Abadie y Gardeazabal (2003) y Abadie, Diamond y Hainmueller (2010); esto es, realizando ejercicios placebos. 


\section{Gráfico 6. Evolución de la Brecha entre el Logaritmo del Salario Real del Grupo de Tratamiento y de Control Sintético. Test Placebo. 2014-2018}

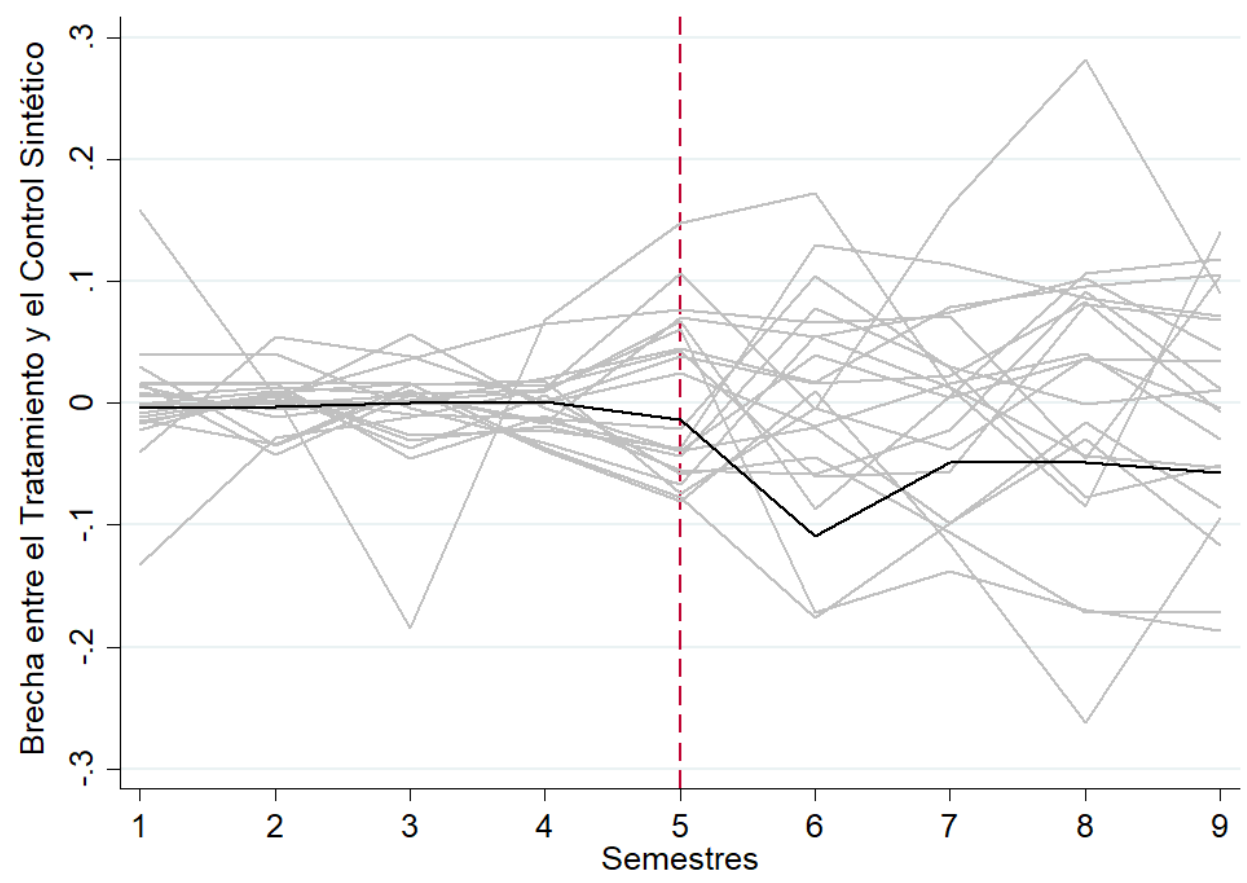

Notas. Fuente: Elaboración propia con base en datos del Departamento Administrativo Nacional de Estadística (DANE). La línea negra denota la brecha cuando en el Grupo de Tratamiento se consideraron a los departamentos de La Guajira y Norte de Santander. Las líneas grises denotan la brecha de ejercicios alternativos considerando como Grupos de Tratamiento al resto de los departamentos no afectados por la migración.

Tal y como se observa en el Gráfico 6 la evolución de la brecha pre-tratamiento y post-tratamiento de los experimentos falsos no sigue una senda como la del grupo real de tratamiento, esto es considerando a La Guajira y Norte de Santander, lo cual evidencia que para cualquiera de las otras regiones no hubo un efecto causal de la apertura de fronteras sobre el salario real.

Adicionalmente en el Gráfico 7 se observa la distribución del ratio entre el Error Cuadrático Medio Predicho (MSPE, por sus siglas en inglés) ${ }^{10}$ post-tratamiento y pre-tratamiento considerando a La Guajira y a Norte de Santander como grupos de tratamiento y, adicionalmente, a los diferentes placebos estimados previamente como medida de qué tan confiable es la estimación realizada mediante MCS.

Se puede apreciar que el ratio en el caso que se considera a La Guajira y Norte de Santander como tratamiento es mucho más grande que cualquiera de los otros ratios calculados como placebos. Esto indica que, sin importar qué otros departamentos consideremos, los únicos con los cuales obtenemos un efecto significativamente elevado sobre el salario real es para el caso en el cual estamos analizando, es decir, aquellos con un incremento significativo en la oferta laboral producto del éxodo migratorio venezolano.

\footnotetext{
${ }^{10}$ El Error Cuadrático Medio Predicho (MSPE) se define como la diferencia al cuadrado entre el outcome del grupo de tratamiento y del grupo de control sintético. Este cálculo se realiza para ambos periodos (pre y post apertura de fronteras) y posteriormente se calcula el ratio entre el MSPE post apertura y el MSPE pre apertura.
} 


\section{Gráfico 7. Ratio MSPE}

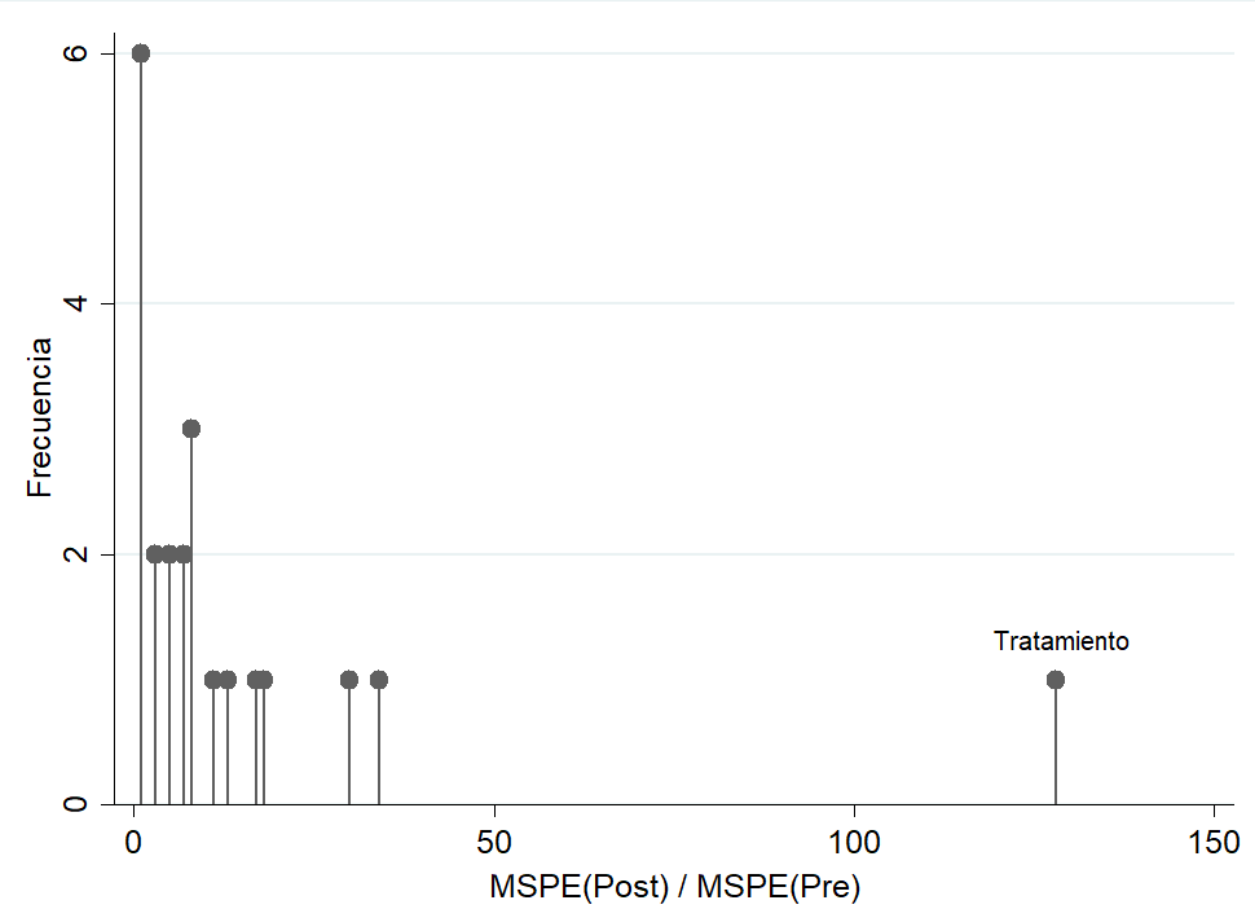

Notas. Fuente: Elaboración propia con base en datos del Departamento Administrativo Nacional de Estadística (DANE).

\section{Grupo de Control Ad-Hoc}

Por otra parte, es posible que la caída del salario real en La Guajira y Norte de Santander se haya debido, más que a un incremento en el flujo migratorio desde Venezuela hacia Colombia, a la crisis macroeconómica en Venezuela. Con lo cual, ante la caída de la capacidad de absorción de la economía venezolana, probablemente haya caído también el comercio internacional con Colombia afectando principalmente a las economías fronterizas, en el caso colombiano, a La Guajira y a Norte de Santander.

Sin embargo, tal y como CEPAL (2017) expone, el PBI de Venezuela ha caído durante cuatro años consecutivos desde el 2013 hasta el 2017, acumulando una contracción del PBI real en esos cuatro años de aproximadamente un $31,9 \%$.

Por lo tanto, si la caída en el salario real analizada en este trabajo post-apertura de las fronteras se hubiera generado a partir de una caída en la demanda por parte de la economía venezolana, entonces el salario real también hubiera caído en los periodos previos al periodo de tratamiento considerado.

Tal y como se observa en el Gráfico 4 en los periodos pre-tratamiento considerados el salario real no mantuvo una tendencia decreciente y el único periodo en el cual se presentó una caída significativa fue en el primer semestre del año 2015, caída que también sufrieron otros departamentos no fronterizos como los considerados en el grupo de control ad-hoc. Por lo tanto, no parecería ser la crisis económica el principal motor de la caída del salario real en La Guajira y Norte de Santander a partir del segundo semestre del año 2016. 
Una forma de estimar la robustez de los resultados obtenidos previamente consiste en suponer que la intervención, en este caso la apertura de las fronteras entre Colombia y Venezuela, se realizó en otro semestre distinto al segundo semestre del año 2016; es decir, realizar experimentos falsos.

Bajo estos supuestos, si se asume que la apertura de la frontera se realizó en cualquiera de estos periodos previos, no se debería obtener ningún efecto sobre el salario real que sea estadísticamente significativo dado que, en la realidad, no hubo un incremento en la oferta laboral.

En caso de obtener un efecto significativo entonces la correlación podría estar dada más por la caída en la demanda agregada del país venezolano, producto de la crisis económica, que por el incremento en la oferta de trabajo. En las Tablas A4 a la A7 del Apéndice se presentan los resultados para cada una de las estimaciones de diferencias en diferencias presentadas previamente asumiendo la fecha de intervención como si hubiera sucedido en los periodos 2015-II, 2015-I y 2014-II.

Los resultados de los experimentos falsos efectivamente sugieren ausencia de efecto estadísticamente significativo cuando se asume una fecha de shock migratorio distinta a la real. Este resultado respalda la validez de la hipótesis propuesta en este trabajo y la potencial causalidad de los efectos estimados previamente entre el incremento en la oferta laboral y la caída del salario real.

Es decir, los resultados permiten inferir que la caída del salario real post-apertura de las fronteras entre Colombia y Venezuela realmente se originó producto de un incremento en la oferta laboral en las regiones fronterizas más que debido a la caída del PBI de Venezuela como consecuencia de la crisis económica.

Adicionalmente, una crítica válida sobre las estimaciones realizadas mediante la metodología de diferencias en diferencias seleccionando un grupo de control ad-hoc, es que los resultados que indican una caída del salario real horario post apertura de las fronteras entre Colombia y Venezuela pueden deberse, únicamente, al grupo de control seleccionado discrecionalmente.

Si bien como medida de robustez se presentaron las estimaciones en agregado empleando el MCS para evitar la discrecionalidad, una medida adicional de robustez de los resultados consistiría en verificar que éstos se mantienen aun cambiando el grupo de control que se selecciona ad-hoc.

Por este motivo, se realizaron las mismas estimaciones de diferencias en diferencias, tanto en agregado como con efectos heterogéneos, pero construyendo un grupo de control alternativo a partir de los departamentos que resultaron con ponderación no nula al implementar MCS (a partir de ahora grupo de control MCS). El grupo de control MCS, por lo tanto, está conformado por los departamentos de Chocó, Magdalena, Nariño y Tolima.

Tal y como se presentó en la Tabla A1, la población venezolana radicada como proporción de la PEA en la mayoría de estos departamentos del grupo de control MCS de acuerdo con el RAMV fue inferior a 1 punto porcentual, excepto para Magdalena para el cual el ratio asciende aproximadamente al $4.5 \%$. Con lo cual, en promedio, el flujo migratorio desde la apertura de las fronteras no tuvo que haber alterado la tendencia de los salarios reales de estos departamentos, permitiendo, de esta manera, que sean usados como grupos de comparación.

Por otro lado, en la Tabla A2 del Apéndice se puede observar el test de tendencias comunes pretratamiento entre el grupo de control MCS y el grupo de tratamiento, el cual permite no rechazar la hipótesis nula de tendencias comunes, validando la selección del grupo de control MCS como una alternativa a nuestro grupo de control ad-hoc presentado en secciones anteriores. 
Los resultados de las estimaciones considerando a La Guajira y Norte de Santander como grupo de tratamiento y al grupo de control MCS se presentan en la Tabla A8 del Apéndice.

De acuerdo con lo que se esperaba, la significatividad estadística y los signos de las estimaciones son consistentes con los resultados obtenidos cuando se empleó al grupo de control ad-hoc, lo cual proporciona robustez a la hipótesis de causalidad entre un incremento de la oferta laboral y una caída de los salarios reales.

Adicionalmente, se observa un mayor efecto, en promedio, del flujo migratorio sobre los salarios reales para la población no calificada en comparación a los calificados, resultado consistente con las estimaciones empleando el grupo de control ad-hoc. Por otro lado, se observa que el efecto estimado sobre la población de ocupados formales es mucho menor en comparación a los ocupados informales y que habría una caída más fuerte en el salario real para los hombres.

Un elemento crucial en los resultados obtenidos es que, si bien la significatividad estadística y el sentido de los efectos son congruentes entre las estimaciones aun cambiando el grupo de control, no sucede lo mismo con la magnitud de los efectos.

Pese a que las magnitudes de los efectos estimados si se compara entre los dos grupos de control toman, en general, valores relativamente cercanos, se puede observar una diferencia en el caso de la estimación agregada dado que empleando el grupo de control ad-hoc la caída en el salario real estimada fue de $9.7 \%$ y empleando el control MCS la caída es de, en promedio, $6.5 \%$.

Esta divergencia en los resultados es, sin embargo, consistente con las observaciones realizadas por Borjas (2017) sobre lo fundamental que resulta la selección de los grupos de control y, por lo tanto, esperable dada la discrecionalidad en la construcción de los grupos de comparación.

\section{COMENTARIOS FINALES}

Una de las preguntas que más discusiones ha generado en el campo de la economía laboral se vincula con la respuesta en el corto plazo del mercado de trabajo ante un aumento significativo de la oferta de trabajadores producto de un flujo migratorio exógeno. El debate lleva más de tres décadas y los resultados obtenidos, a partir de diferentes metodologías y marcos conceptuales, no dejan de ser ambiguos ni parecerían ir en un sentido de convergencia.

En el presente trabajo se presentaron estimaciones del potencial impacto causal sobre el salario real del flujo migratorio de venezolanos hacia Colombia debido a la reapertura de la frontera entre Colombia y Venezuela en el 2016 y, adicionalmente, de la crisis económica y social del país venezolano que lleva aproximadamente media década.

Los resultados evidencian una caída significativa del salario real horario ( $9.7 \%$ aproximadamente) en aquellas regiones en las que el flujo migratorio fue muy importante con relación a la población económicamente activa.

Las estimaciones parecerían indicar una caída del salario real más fuerte para los hombres y para los trabajadores en condiciones de informalidad laboral. Adicionalmente, el efecto causal generado por la migración sobre la variable precio del mercado laboral sería mucho más fuerte en aquellos individuos de la población con menor nivel de calificación, resultado que es consistente con la literatura de downgrading.

Por otro lado, los resultados presentados en este trabajo son robustos a diferentes especificaciones y análisis realizados que permiten asegurar el mecanismo causal entre el flujo migratorio como shock de oferta laboral y la caída en el salario real. 
A la luz de estos resultados y considerando la vulnerabilidad social y económica de las regiones que se han visto más fuertemente afectadas por la caída del salario real, producto del flujo migratorio de venezolanos, es importante la implementación de políticas públicas que, de manera coordinada, mitiguen el efecto del shock de oferta laboral sobre los salarios reales.

Bajo el marco analítico canónico implementado para estimar los efectos causales del presente trabajo, la caída del salario real post shock de oferta laboral es un resultado de equilibrio parcial que presupone que el resto de las variables del mercado laboral se mantienen constantes.

Dados los resultados se puede asumir entonces que tal y como Borjas (2003) lo afirma, la demanda de trabajo en estas regiones tiene pendiente negativa. Con lo cual, ante una oferta de trabajo que no es infinitamente elástica, políticas públicas que impulsen una mayor demanda de trabajo permitirían garantizar que el salario real caiga en menor proporción luego del incremento en la oferta de trabajo debido a la llegada de venezolanos a tierras colombianas.

Un escenario positivo para la población fronteriza del lado colombiano sería uno bajo el cual, tal y como Card (1990) argumentaba de manera preliminar para Miami, las regiones de Colombia que recibieron mano de obra venezolana tuvieran las capacidades de absorción de empleo de manera tal que el salario real no caiga y se fortalezca la economía.

Sin embargo, dada la incapacidad del mercado laboral de absorber la mano de obra, es imperante la intervención estatal mediante un programa de generación de empleo y/o de impulso de la demanda agregada que permita amortiguar las consecuencias económicas de la migración.

De continuar la tendencia en la caída del salario real principalmente de la población en condiciones de informalidad y/o de menor calificación, se esperaría que, en el mediano plazo, los efectos sobre la desigualdad sean significativos profundizando el deterioro social e incrementando la inequidad y la pobreza tanto intra-regionalmente como a nivel nacional.

Suponiendo una división de la población entre mano de obra calificada y no calificada se esperaría que, de mantenerse la tendencia estimada en este trabajo, el ingreso laboral relativo del primer grupo con relación al segundo aumente considerablemente generando una brecha social difícil de revertir. Este tipo de análisis, sin embargo, excede el alcance de este trabajo y deberá ser objetivo de futuras investigaciones. 


\section{REFERENCIAS BIBLIOGRÁFICAS}

Abadie, A., Diamond, A., \& Hainmueller, J. (2010). Synthetic control methods for comparative case studies: Estimating the effect of California's tobacco control program. Journal of the American statistical Association, 105(490), 493-505.

Abadie, A., \& Gardeazabal, J. (2003). The economic costs of conflict: A case study of the Basque Country. American economic review, 93(1), 113-132.

Alix-Garcia, J., \& Saah, D. (2009). The effect of refugee inflows on host communities: evidence from Tanzania. The World Bank Economic Review, 24(1), 148-170.

Altonji, J. G., \& Card, D. (1991). The effects of immigration on the labor market outcomes of lessskilled natives. In Immigration, trade, and the labor market (pp. 201-234). University of Chicago Press.

Angrist, J. D., \& Kugler, A. D. (2003). Protective or counter-productive? Labour market institutions and the effect of immigration on EU natives. The Economic Journal, 113(488).

Borjas, G. J. (1985). Assimilation, changes in cohort quality, and the earnings of immigrants. Journal of labor Economics, 3(4), 463-489.

Borjas, G. J. (2003). The labor demand curve is downward sloping: Reexamining the impact of immigration on the labor market. The quarterly journal of economics, 118(4), 1335-1374.

Borjas, G. J. (2017). The wage impact of the Marielitos: A reappraisal. ILR Review, 70(5), 10771110.

Card, D. (1990). The impact of the Mariel boatlift on the Miami labor market. ILR Review, 43(2), 245-257.

Carrington, W. J., \& de Lima, P. (1994). Large-scale immigration and labor markets: an analysis of the retornados and their impact on Portugal. Johns Hopkins $U$.

Comisión Económica para América Latina, \& el Caribe. (2017). Balance Preliminar de las Economías de América Latina y el Caribe 2017. United Nations Publications.

Dustmann, C., \& Preston, I. (2012). Comment: Estimating the effect of immigration on wages. Journal of the European Economic Association, 10(1), 216-223.

Dustmann, C., Frattini, T., \& Preston, I. P. (2013). The effect of immigration along the distribution of wages. Review of Economic Studies, 80(1), 145-173.

Dustmann, C., Schönberg, U., \& Stuhler, J. (2016). The impact of immigration: Why do studies reach such different results? Journal of Economic Perspectives, 30(4), 31-56.

Friedberg, R. M. (2001). The impact of mass migration on the Israeli labor market. The Quarterly Journal of Economics, 116(4), 1373-1408.

Gardeazabal, J. (2010). Methods for measuring aggregate costs of conflict.

Garganta, S., \& Gasparini, L. (2012). El impacto de un programa social sobre la informalidad laboral: el caso de la AUH en Argentina (No. 133). Documento de Trabajo.

Hunt, J. (1992). The impact of the 1962 repatriates from Algeria on the French labor market. ILR Review, 45(3), 556-572. 
Lozano, F. A., \& Steinberger, M. D. (2012). Empirical methods in the economics of international immigration. Handbook of Research Methods in Migration, 163-185.

Malaeb, B., \& Wahba, J. (2018). Impact of Refugees on Immigrants' Labor Outcomes. In Economic Research Forum Working Paper Series (Forthcoming). Cairo, Egypt.

Peri, G., \& Yasenov, V. (2018). The labor market effects of a refugee wave: Synthetic control method meets the Mariel boatlift. Journal of Human Resources, 0217_8561R1.

Tumen, S. (2016). The economic impact of Syrian Refugees on host countries: QuasiExperimental evidence from Turkey. American Economic Review, 106(5), 456-60. 


\section{APÉNDICE}

Tabla A1. Flujo Migratorio Colombia como Porcentaje de la Población Económica Activa (2016-2018)

\begin{tabular}{lcccc}
\hline \hline Departamento & $\begin{array}{c}\text { Participación } \\
\text { hasta 2017-I }\end{array}$ & $\begin{array}{c}\text { Participación } \\
\text { hasta 2017-II }\end{array}$ & $\begin{array}{c}\text { Participación } \\
\text { hasta } \\
\text { 2018-I RAMV }\end{array}$ & $\begin{array}{c}\text { Diferencia } \\
\text { (p.p) }\end{array}$ \\
\hline Amazonas & $0.65 \%$ & $1.03 \%$ & $0.03 \%$ & -1.00 \\
Antioquia & $0.99 \%$ & $1.86 \%$ & $0.53 \%$ & -1.33 \\
Arauca & $66.80 \%$ & $69.75 \%$ & $63.65 \%$ & -6.10 \\
Atlántico & $3.35 \%$ & $6.16 \%$ & $2.72 \%$ & -3.44 \\
Bogotá D.C. & $4.18 \%$ & $8.60 \%$ & $0.75 \%$ & -7.85 \\
Bolívar & $1.81 \%$ & $2.71 \%$ & $2.00 \%$ & -0.71 \\
Boyacá & $0.18 \%$ & $0.34 \%$ & $0.50 \%$ & 0.16 \\
Caldas & $0.10 \%$ & $0.21 \%$ & $0.22 \%$ & 0.01 \\
Caquetá & $0.01 \%$ & $0.02 \%$ & $0.01 \%$ & 0.00 \\
Casanare & $0.85 \%$ & $1.63 \%$ & $5.27 \%$ & 3.64 \\
Cauca & $0.02 \%$ & $0.04 \%$ & $0.13 \%$ & 0.09 \\
Cesar & $1.40 \%$ & $2.01 \%$ & $3.61 \%$ & 1.59 \\
Choco & $0.04 \%$ & $0.07 \%$ & $0.09 \%$ & 0.02 \\
Córdoba & $0.16 \%$ & $0.22 \%$ & $0.61 \%$ & 0.38 \\
Cundinamarca & $0.19 \%$ & $0.32 \%$ & $0.60 \%$ & 0.28 \\
Guainía & $19.78 \%$ & $22.24 \%$ & $16.67 \%$ & -5.57 \\
Guaviare & $0.12 \%$ & $0.25 \%$ & $0.26 \%$ & 0.02 \\
Huila & $0.04 \%$ & $0.07 \%$ & $0.21 \%$ & 0.14 \\
La Guajira & $8.65 \%$ & $15.67 \%$ & $12.35 \%$ & -3.32 \\
Magdalena & $1.57 \%$ & $2.50 \%$ & $4.48 \%$ & 1.98 \\
Meta & $0.23 \%$ & $0.39 \%$ & $0.51 \%$ & 0.12 \\
Nariño & $6.45 \%$ & $21.22 \%$ & $0.09 \%$ & -21.14 \\
Norte de Santander & $6.70 \%$ & $11.66 \%$ & $10.26 \%$ & -1.39 \\
Putumayo & $0.49 \%$ & $0.81 \%$ & $4.19 \%$ & 3.38 \\
Quindío & $0.22 \%$ & $0.45 \%$ & $0.31 \%$ & -0.14 \\
Risaralda & $0.28 \%$ & $0.54 \%$ & $0.41 \%$ & -0.12 \\
Santander & $1.19 \%$ & $2.43 \%$ & $0.74 \%$ & -1.68 \\
Sucre & $0.27 \%$ & $0.38 \%$ & $1.01 \%$ & 0.63 \\
Tolima & $0.08 \%$ & $0.16 \%$ & $0.18 \%$ & 0.02 \\
Valle del Cauca & $0.45 \%$ & $0.84 \%$ & $0.52 \%$ & -0.31 \\
Vaupés & $0.07 \%$ & $281.10 \%$ & $18.88 \%$ & -262.22 \\
Vichada & & & & \\
\hline & & & - & \\
\hline
\end{tabular}

Notas. Fuente: Elaboración propia con base en datos del Departamento Administrativo Nacional de Estadística (DANE), de la Unidad Administrativa Especial Migración Colombia (UAEMC) y del Registro Administrativo de Migrantes Venezolanos en Colombia (RAMV). La población económicamente activa de Amazonas y Casanare corresponden a la del 2015. Para el resto de los departamentos se consideró la PEA del año 2016. Para el caso de los datos del RAMV se consideró únicamente a la población mayor a 12 años. 
Tabla A2. Tests de Tendencias Iguales Pre-Tratamiento

\begin{tabular}{|c|c|c|}
\hline & Estadístico F & P-Valor \\
\hline Control Ad-Hoc & 0.34 & 0.8475 \\
\hline Control MCS & 0.33 & 0.8557 \\
\hline \multicolumn{3}{|c|}{$\begin{array}{l}\text { Notas. Fuente: Elaboración propia con base en datos de la GEIH del DANE. P Valore } \\
\text { y Estadísticos } \mathrm{F} \text { asociados a las estimaciones obtenidas de un modelo de variabl } \\
\text { dependiente igual al logaritmo del salario horario real, una constante, una dummy d } \\
\text { tratamiento, dummies por periodo y la interacción entre las dummies de periodo y d } \\
\text { tratamiento. Se consideraron únicamente los periodos pretratamiento. Se aplicó u } \\
\text { test } \mathrm{F} \text { en el cual bajo hipótesis nula las interacciones de las regresiones era } \\
\text { conjuntamente iquales a } 0 \text {. }\end{array}$} \\
\hline
\end{tabular}

Tabla A3. Ponderaciones de los Departamentos mediante Método de Control Sintético

\begin{tabular}{cc}
\hline \hline Departamento & Ponderación \\
\hline Antioquia & $0.0 \%$ \\
Atlántico & $0.0 \%$ \\
Bogotá & $0.0 \%$ \\
Bolívar & $0.0 \%$ \\
Boyacá & $0.0 \%$ \\
Caldas & $0.0 \%$ \\
Caquetá & $0.0 \%$ \\
Cauca & $0.0 \%$ \\
Cesar & $0.0 \%$ \\
Córdoba & $0.0 \%$ \\
Chocó & $17.7 \%$ \\
Huila & $0.0 \%$ \\
Magdalena & $27.5 \%$ \\
Meta & $0.0 \%$ \\
Nariño & $5.7 \%$ \\
Quindío & $0.0 \%$ \\
Risaralda & $0.0 \%$ \\
Santander & $0.0 \%$ \\
Sucre & $0.0 \%$ \\
Tolima & $49.1 \%$ \\
Valle del Cauca & $0.0 \%$ \\
\hline
\end{tabular}

Notas. Fuente: Elaboración propia con base en datos del DANE. Las variables explicativas que se emplearon para el método de Control Sintético son la proporción de empleado por rama de actividad económica, la tasa de informalidad laboral, el promedio de años de educación del departamento, la proporción de trabajadores calificados, la tasa de desempleo y el logaritmo del salario horario de periodos pretratamiento específicos. 
Tabla A4. Estimaciones Placebos Agregadas

Variable Dependiente: Logaritmo del Salario Horario Real

\begin{tabular}{lccc}
\hline & \multicolumn{3}{c}{ Control Ad-Hoc } \\
\hline & $2015-$ II & $2015-I$ & $2014-I I$ \\
Efecto Migración & $\mathbf{0 . 0 1 1 1}$ & $\mathbf{0 . 0 0 8 6 0}$ & $\mathbf{0 . 0 0 0 7 3 8}$ \\
& $\mathbf{( 0 . 0 2 3 1 )}$ & $\mathbf{( 0 . 0 2 0 2 )}$ & $\mathbf{( 0 . 0 2 1 2 )}$ \\
Observaciones & 20,400 & 20,400 & $\mathbf{2 0 , 4 0 0}$ \\
$\mathrm{R}^{2}$ & 0.356 & 0.356 & 0.356 \\
\hline Controles Individuales & Sí & Sí & Sí \\
Controles Agregados y Temporales & Sí & Sí & Sí \\
\hline
\end{tabular}

Notas. Errores estándar robustos y clusterizados a nivel periodo-departamento entre paréntesis. Las estrellas ${ }^{*},{ }^{* *} \mathrm{y}^{* * *}$ denotan significatividad al 10,5 y 1 por ciento. Las observaciones corresponden al periodo 2014-2018 con datos de junio y diciembre de cada año hasta la primera mitad del 2018. Se restringió la muestra para periodos previos a junio de 2016 inclusive. Los controles individuales incluyen características vinculadas con el sexo del individuo, su nivel de educación lineal y al cuadrado, la edad lineal y al cuadrado, si es un empleado formal o no y el estado civil. Los controles agregados y temporales se vinculan con dummies regionales, temporales y de rama de actividad del individuo. Fuente: Elaboración propia con base en la GEIH.

Tabla A5. Estimaciones Placebos por Calificación.

Variable Dependiente: Logaritmo del Salario Horario Real

\begin{tabular}{lccc}
\hline & \multicolumn{3}{c}{ Control Ad-Hoc } \\
\hline & $2015-I I$ & $2015-I$ & $2014-I I$ \\
\hline \multirow{3}{*}{ Efecto Migración } & & & \\
& $\mathbf{- 0 . 0 1 1 2}$ & $\mathbf{- 0 . 0 0 9 5 0}$ & $\mathbf{0 . 0 1 9 6}$ \\
Efecto Migración×Califica & $\mathbf{( 0 . 0 4 2 5 )}$ & $\mathbf{( 0 . 0 3 6 1 )}$ & $\mathbf{( 0 . 0 4 1 5 )}$ \\
& $\mathbf{0 . 0 3 8 9}$ & $\mathbf{0 . 0 3 2 7}$ & $\mathbf{- 0 . 0 2 4 9}$ \\
Observaciones & $\mathbf{( 0 . 0 3 8 5 )}$ & $\mathbf{( 0 . 0 3 2 6 )}$ & $\mathbf{( 0 . 0 3 8 2 )}$ \\
$\mathrm{R}^{2}$ & $\mathbf{2 0 , 4 0 0}$ & 20,400 & $\mathbf{2 0 , 4 0 0}$ \\
\hline Controles Individuales & 0.358 & 0.358 & 0.358 \\
Controles Agregados y Temporales & Sí & Sí & Sí \\
\hline
\end{tabular}

Notas. Errores estándar robustos y clusterizados a nivel periodo-departamento entre paréntesis. Las estrellas ${ }^{*},{ }^{* *} y^{* * *}$ denotan significatividad al 10,5 y 1 por ciento. Las observaciones corresponden al periodo 2014-2018 con datos de junio y diciembre de cada año hasta la primera mitad del 2018. Se restringió la muestra para periodos previos a junio de 2016 inclusive. Los controles individuales incluyen características vinculadas con el sexo del individuo, su nivel de educación lineal y al cuadrado, la edad lineal y al cuadrado, si es un empleado formal o no y el estado civil. Los controles agregados y temporales se vinculan con dummies regionales, temporales y de rama de actividad del individuo. Fuente: Elaboración propia con base en la GEIH. 
Tabla A6. Estimaciones Placebos por Sexo.

Variable Dependiente: Logaritmo del Salario Horario Real

\begin{tabular}{lccc}
\hline & \multicolumn{3}{c}{ Control Ad-Hoc } \\
\hline & $2015-$ II & $2015-I$ & $2014-I I$ \\
Efecto Migración & & & \\
& $\mathbf{- 0 . 0 0 3 2 4}$ & $\mathbf{0 . 0 0 8 2 5}$ & $\mathbf{- 0 . 0 1 5 2}$ \\
Efecto Migración ×Mujer & $\mathbf{( 0 . 0 2 9 8 )}$ & $\mathbf{( 0 . 0 2 6 3 )}$ & $\mathbf{( 0 . 0 2 9 8 )}$ \\
& $\mathbf{0 . 0 2 9 7}$ & $\mathbf{0 . 0 0 0 8 0 2}$ & $\mathbf{0 . 0 3 4 0}$ \\
Observaciones & $\mathbf{( 0 . 0 2 8 8 )}$ & $\mathbf{( 0 . 0 3 2 6 )}$ & $\mathbf{( 0 . 0 3 4 3 )}$ \\
$\mathrm{R}^{2}$ & 20,400 & 20,400 & 20,400 \\
\hline Controles Individuales & 0.357 & 0.357 & 0.357 \\
Controles Agregados y Temporales & Sí & Sí & Sí \\
\hline
\end{tabular}

Notas. Errores estándar robustos y clusterizados a nivel periodo-departamento entre paréntesis. Las estrellas ${ }^{*},{ }^{* *} \mathrm{y}^{* * *}$ denotan significatividad al 10, 5 y 1 por ciento. Las observaciones corresponden al periodo 2014-2018 con datos de junio y diciembre de cada año hasta la primera mitad del 2018. Se restringió la muestra para periodos previos a junio de 2016 inclusive. Los controles individuales incluyen características vinculadas con el nivel de educación lineal y al cuadrado, la edad lineal y al cuadrado, si es un empleado formal o no y el estado civil. Los controles agregados y temporales se vinculan con dummies regionales, temporales y de rama de actividad del individuo. Fuente: Elaboración propia con base en la GEIH.

Tabla A7. Estimaciones Placebos por Formalidad Laboral

Variable Dependiente: Logaritmo del Salario Horario Real

\begin{tabular}{lccc}
\hline & \multicolumn{4}{c}{ Control Ad-Hoc } \\
\hline & $2015-I I$ & $2015-I$ & $2014-I I$ \\
Efecto Migración & & & \\
& $-\mathbf{0 . 0 0 1 7 4}$ & $-\mathbf{0 . 0 1 4 0}$ & $\mathbf{0 . 0 1 0 2}$ \\
Efecto Migración×Formal & $\mathbf{( 0 . 0 3 1 4 )}$ & $\mathbf{( 0 . 0 3 1 8 )}$ & $\mathbf{( 0 . 0 3 2 6 )}$ \\
& $\mathbf{0 . 0 4 9 1}$ & $\mathbf{0 . 0 5 6 8}$ & $\mathbf{- 0 . 0 1 1 2}$ \\
Observaciones & $\mathbf{( 0 . 0 3 1 2 )}$ & $\mathbf{( 0 . 0 3 5 9 )}$ & $\mathbf{( 0 . 0 4 1 5 )}$ \\
$\mathrm{R}^{2}$ & $\mathbf{2 0 , 4 0 0}$ & 20,400 & $\mathbf{2 0 , 4 0 0}$ \\
\hline Controles Individuales & 0.357 & 0.357 & 0.357 \\
Controles Agregados y Temporales & Sí & Sí & Sí \\
\hline
\end{tabular}

Notas. Errores estándar robustos y clusterizados a nivel periodo-departamento entre paréntesis. Las estrellas ${ }^{*},{ }^{* *} y^{* * *}$ denotan significatividad al 10,5 y 1 por ciento. Las observaciones corresponden al periodo 2014-2018 con datos de junio y diciembre de cada año hasta la primera mitad del 2018. Se restringió la muestra para periodos previos a junio de 2016 inclusive. Los controles individuales incluyen características vinculadas con el sexo del individuo, su nivel de educación lineal y al cuadrado, la edad lineal y al cuadrado y el estado civil. Los controles agregados y temporales se vinculan con dummies regionales, temporales y de rama de actividad del individuo. Fuente: Elaboración propia con base en la GEIH. 
Tabla A8. Estimaciones de Robustez

Variable Dependiente: Logaritmo del Salario Horario Real

\begin{tabular}{lcccc}
\hline & \multicolumn{4}{c}{ Grupo de Control MCS } \\
\hline & Agregado & Calificación & Sexo & Formalidad \\
\hline Efecto Migración & & & & \\
& $-0.0647^{* * *}$ & $-0.105^{* * *}$ & $-0.0953^{* * *}$ & $-0.0842^{* * *}$ \\
Efecto Migración×Calificado & $(0.0175)$ & $(0.0265)$ & $(0.0200)$ & $(0.0216)$ \\
Efecto Migración×Mujer & & $0.0658^{* *}$ & & \\
& & $(0.0257)$ & & \\
Efecto Migración×Formal & & & $0.0633^{* *}$ & \\
Observaciones & & & $(0.0274)$ & \\
$\mathrm{R}^{2}$ & & & & $0.0728^{* * *}$ \\
\hline Controles Individuales & 37,557 & 37,557 & 37,557 & 37,557 \\
Controles Agregados y Temporales & 0.368 & 0.371 & 0.369 & 0.369 \\
\hline
\end{tabular}

Notas. Errores estándar robustos y clusterizados a nivel periodo-departamento entre paréntesis. Las estrellas *, ** y *** denotan significatividad al 10, 5 y 1 por ciento. Las observaciones corresponden al periodo 2014-2018 con datos de junio y diciembre de cada año hasta la primera mitad del 2018. Los controles individuales incluyen características vinculadas con el sexo del individuo, su nivel de educación lineal y al cuadrado, la edad lineal y al cuadrado, si es un empleado formal o no y el estado civil. Los controles agregados y temporales se vinculan con dummies regionales, temporales y de rama de actividad del individuo. Fuente: Elaboración propia con base en la GEIH. 